This is the accepted manuscript version of the following manuscript:

Laura Espinar-Barranco, Pilar Luque-Navarro, Maja A. Strnad, Pilar HerreroFoncubierta, Luis Crovetto, Delia Miguel, Maria D. Giron, Angel Orte, Juan M. Cuerva, Rafael Salto, Jose M. Alvarez-Pez, Jose M. Paredes, A solvatofluorochromic siliconsubstituted xanthene dye useful in bioimaging, Dyes and Pigments, Volume 168, 2019, Pages 264-272, ISSN 0143-7208, https://doi.org/10.1016/j.dyepig.2019.04.024.

This article may be used for non-commercial purposes in accordance with Elsevier Sharing Policies.

This accepted manuscript has been made available after a 24-month embargo period.

Supplementary Materials accompanying this article can be found on-line at the publisher's site. 


\section{A solvatofluorochromic silicon-substituted xanthene dye useful in bioimaging}

Laura Espinar-Barranco ${ }^{a}$, Pilar Luque-Navarro ${ }^{a}$, Maja A. Strnad ${ }^{a}$, Pilar Herrero-Foncubierta ${ }^{a, c}$, Luis Crovetto ${ }^{a}$, Delia Miguel ${ }^{a}$, Maria D. Giron ${ }^{b}$, Angel Orte*a, Juan M. Cuerva ${ }^{c}$, Rafael Salto ${ }^{b}$, Jose M. Alvarez-Pez ${ }^{\mathrm{a}}$, Jose M. Paredes*a

[a] Departamento de Fisicoquimica. Facultad de Farmacia. Unidad de Excelencia en Quimica Aplicada a Biomedicina y Medioambiente (UEQ). Universidad de Granada. Campus Cartuja, 18071 Granada (Spain) E-mail: jmparedes@ugr.es; angelort@ugr.es

[b] Departamento de Bioquímica y Biología Molecular. Facultad de Farmacia. Unidad de Excelencia en Quimica Aplicada a Biomedicina y Medioambiente (UEQ). Universidad de Granada. Campus Cartuja, 18071 Granada (Spain)

[c] Departamento de Química Orgánica. Facultad de Ciencias. Unidad de Excelencia en Quimica Aplicada a Biomedicina y Medioambiente (UEQ). C. U. Fuentenueva s/n, 18071 Granada (Spain)

\section{$\underline{\text { Abstract }}$}

In this work, we have performed an in-depth study of the photophysics and solvatofluorochromism of a red-emitting Si-xanthene dye, an analog of Tokyo Magenta (TM) historically developed by Egawa et al (Chem. Commun. 2011, 47, 4162-4164). The results show a strong dependency of the emission properties of 2-Me-4-OMe-TM on the polarity of the solvent. For instance, the dye exhibited an increase in its fluorescence lifetime with the decrease in solvent polarity. Therefore, in this work, this spectral behavior has been used as a new approach for determining the intracellular microenvironment polarity through the measurement of its fluorescence lifetime by Fluorescence Lifetime Imaging Microscopy (FLIM). Our experiments confirmed the ability of the dye to detect changes in polarity between different intracellular compartments. 
Keywords: Hetero-xanthene dyes; Solvatofluorochromism; Fluorescent dyes; Biosensors; Photophysics

\section{Introduction}

In the last few years, chemical modifications of the prototype fluorescein scaffold have produced a great variety of new fluorescent xanthene-based dyes with tailored photophysical properties. ${ }^{[1]}$ However, all these derivatives present a common drawback: they exhibit absorption and emission wavelength maxima in the green region of the visible spectral range. On the other hand, the replacement of the oxygen atom at the $10^{\prime}$ position of the xanthene moiety by a silicon atom has a significant impact on the photophysical properties, thus producing a usually desirable red-shift in their absorption and emission maxima. ${ }^{[2]}$ For reviews, see can be found also. ${ }^{[3]}$ These new derivatives are now synthetically accessible, and more importantly, their applications in biosensing and bioimaging has been demonstrated. ${ }^{[4]}$ Within this context, our research group has recently described the presence of an excited-state proton transfer (ESPT) reaction promoted by the species of phosphate buffer in these fluorophores. ${ }^{[5]}$ When an adequate proton donor/acceptor pair, as a phosphate buffer species, is present, this ESPT reaction produces a change in the fluorescence lifetime that depends on the total phosphate concentration and is therefore a useful method for phosphate detection. Remarkably, silicon-containing fluorophores are spontaneously incorporated into cells and can be used to monitor intracellular phosphate fluxes using fluorescence lifetime imaging microscopy (FLIM). ${ }^{[6]}$ FLIM is an excellent approach for studying biological systems because of the inherent advantages of this technique, such as fluorophore concentration independence or easy autofluorescence avoidance. ${ }^{[7]}$ FLIM incorporates the advantages of fluorescence probes but uses fluorescence lifetime as a sensing parameter. In FLIM, intensity images are recorded together with fluorescence lifetime, allowing a multiparameter analysis of data. FLIM-based intracellular probes have been successfully used in the determination of $\mathrm{pH}^{[8]}{ }^{[8}$ calcium ${ }^{[9]}$ and 
phosphate $\mathrm{e}^{[6]}$ ions, temperature ${ }^{[10]}$ and microviscosity. ${ }^{[11]}$ The incorporation of the new family of fluorescein red derivatives in FLIM methodology thus opens new opportunities in the research of biological systems, where this technique is preferentially used. In this context, we designed, synthesized and described a modified fluorophore, ${ }^{[12]}$ 7-hydroxy-10(4-methoxy-2methylphenyl)-5,5-dimethyldibenzo[b,e]silin-3(5H)-one (2-Me-4-OMe-TM), which could act as a dual multiplexed intracellular detector of biothiols and phosphate ions. ${ }^{[12]}$ However, although the decrease in the fluorescence lifetime was consistent and allowed the phosphate flux in the cell to be monitored, an in-depth study of the buffer-mediated ESPT reaction was lacking. In this work, we unraveled the full excited-state behavior of 2-Me-4-OMe-TM. With this information in hand, we found that when this dye was employed in intracellular FLIM imaging, the fluorescence lifetime was less sensitive towards phosphate ions than the behavior predicted by the ESPT reaction. For this reason, a deep study has been conducted to unravel the reasons for this anomalous behavior. We considered that a combination of different effects might influence the dynamics of the excited state of 2-Me-4-OMe-TM. Because cytoplasm is a complex matrix with multiple compartments and macromolecules, we evaluated whether dye-macromolecule interactions or changes in the polarity of the microenvironment surrounding the dye could change the photophysical properties of 2-Me-4OMe-TM.

One of the reasons behind this anomaly could be the presence of solvatochromic effects in the red fluorescent 2-Me-4-OMe-TM dye. Solvatochromism is the characteristic of a chromophore that undergoes a shift in its absorption and/or emission wavelengths when the substance is dissolved in solvents with different polarities. For shifts in the emission spectra we refer to this phenomenon as solvatofluorochromism. This effect has multiple applications in biological and biomedical research to study local polarity in biological structures, changes in the protein conformation or biomolecule interactions. ${ }^{[13]}$ Despite its wide use, solvatochromism is an unclear phenomenon $^{[14]}$ in which different interactions and dynamic processes are involved. 
These interactions are divided into two types: specific (e.g., hydrogen bonding, charge shift, probe-probe interactions, conformational changes) and nonspecific (when the shift is produced by the nature of the solvent, characterized by the dielectric constant and the refractive index $)^{[15]}$

To shed light on the solvatofluorochromic behavior of 2-Me-4-OMe-TM, we have now carried out an in-depth photophysical study of this red dye using different solvents and different correlation approaches: the Lippert-Mataga equation, the Catalan and $E_{T}(30)$ analysis. The proper use of the solvatofluorochromic behavior of the dye could reveal the presence of the probe in environments with different polarities. To check this assertion, we have also incorporated the probe into a micellar system. We also found a strong solvatofluorochromic effect between protic and aprotic solvents due to specific interactions between the probe and solvent molecules. With all these data, we have reached a deeper understanding of the excited-state dynamics of the hetero-xanthene dye, facilitating its efficient use as a FLIM probe.

\section{Materials and methods}

\section{$\underline{2.1 \text { Instrumentation }}$}

UV-visible absorption spectrophotometry and steady-state fluorimetry were carried out using a Lambda 650 UV-visible spectrophotometer (PerkinElmer, Waltham, MA, USA) and a Jasco FP8300 spectrofluorometer (Jasco, Tokyo, Japan), respectively. Fluorescence lifetime decays were recorded by the single-photon timing method using a FluoTime 200 fluorometer (PicoQuant GmbH, Berlin, Germany). The excitation source was a pulsed diode laser, LDH-530 (PicoQuant), operated at a repetition rate of $20 \mathrm{MHz}$. Fluorescence decay histograms were collected at 600,605 and $610 \mathrm{~nm}$ over 1320 channels, with a time increment per channel of 37 ps. Histograms of the instrument response functions (using a LUDOX scatterer) and sample decays were recorded until they reached $2 \times 10^{4}$ counts in the peak channel. The fluorescence 
decay traces were globally analyzed using an iterative deconvolution method with exponential models using FluoFit software (PicoQuant).

\section{$\underline{2.2 \text { Cell culture }}$}

HEK293 cells (ATCC: CRL-1573) were provided by the Cell Culture Facility, University of Granada. HEK cells were grown in Dulbecco's modified Eagle's medium (DMEM) supplemented with $10 \%(\mathrm{v} / \mathrm{v}) \mathrm{FBS}, 2 \mathrm{mM}$ glutamine, $100 \mathrm{U} / \mathrm{mL}$ penicillin and $0.1 \mu \mathrm{g} / \mathrm{mL}$ streptomycin in a humidified $5 \% \mathrm{CO}_{2}$ incubator.

For the FLIM microscopy experiments, cells were seeded onto $25 \mathrm{~mm}$ coverslips in 6-well plates at a density of $22.5 \times 10^{4}$ cells/well. For their visualization, the samples were washed three times using phosphate buffered saline (PBS) and bathed into Krebs-Ringer phosphate (KRP) buffer composed of $118 \mathrm{mM} \mathrm{NaCl}, 5 \mathrm{mM} \mathrm{KCl}, 1.3 \mathrm{mM} \mathrm{CaCl}_{2}, 1.2 \mathrm{mM} \mathrm{KH}_{2} \mathrm{PO}_{4}$ and $30 \mathrm{mM}$ HEPES with $2 \times 10^{-7} \mathrm{M}$ of 2-Me-4-OMe-TM.

\subsection{Species-associated emission spectra (SAEMS)}

Fluorescence decay traces were collected from 546 to $670 \mathrm{~nm}$, with a $\Delta \lambda$ of $2 \mathrm{~nm}$, in a discrete period of time, subsequently corrected and then normalized to the corresponding steady-state emission spectrum. The equation used to calculate the SAEMS for each detected species, $i$, was the following:

$$
\operatorname{SAEMS}\left(\lambda_{e m}\right)_{i}=\frac{A_{i, \lambda_{e m}} \times \tau_{i}}{\sum\left(A_{i, \lambda_{e m}} \times \tau_{i}\right)} \times I_{s s, \lambda_{e m}}
$$

where $A_{i, \lambda_{e m}}$ is the amplitude of species $i$ at the emission wavelength $\lambda_{\text {em }}, \tau_{i}$ is the fluorescence lifetime of species $i$, and $I_{s s, \lambda_{e m}} i s$ the steady-state emission spectrum.

\subsection{FLIM bioimaging}


FLIM images were recorded on a MicroTime 200 fluorescence-lifetime microscope system (PicoQuant) using the same excitation source described earlier. The light beam passed through a dichroic mirror (532dcxr, Chroma) and through the oil immersion objective (1.4 NA, 100x) specific to an inverted microscope system (IX-71, Olympus). The fluorescence emitted light was collected back through the objective, filtered by a long-pass filter (550LP, AHF/Chroma), and directed to a $75-\mu \mathrm{m}$ confocal aperture. The light was transmitted to a $630 / 60$ bandpass filter (Thorlabs) and focused on single-photon avalanche diodes (SPCM-AQR 14, Perkin Elmer). The data were collected by a TimeHarp 200 TCSPC module (PicoQuant), and raw fluorescence lifetime images were acquired by a scanner with $512 \times 512$ pixel resolution. To obtain the fitted FLIM images, a spatial binning of $2 \times 2$ pixels was performed with SymphoTime software, and the matrix data were exported and analyzed by a home-coded Fiji ([Fiji Is Just] ImageJ) program. ${ }^{[16]}$

\section{Results and discussion}

\section{$\underline{3.1 \text { Solvatofluorochromism. }}$}

The dye 2-Me-4-OMe-TM was prepared as described in the Appendix A (Scheme A1). 2-Me-4OMe-TM was dissolved in 16 different solvents, and we measured its absorption spectra (Figure A1), steady-state fluorescence spectra (Figures A2 and A3), quantum yields (Table A1), and fluorescence lifetimes (Table A2). The comparison of the spectra showed important changes in the photophysical properties of the dye dissolved in different solvents. Interestingly, according to the dye's spectroscopic features, we found two different groups of solvents. In the first group, the dye's absorption was characterized by a band centered between 420 and $446 \mathrm{~nm}$; in the second group, the dye presented two absorption bands, one centered between 434 and $474 \mathrm{~nm}$ and the other between 592 and $613 \mathrm{~nm}$. The main feature of most of the solvents in which the dye displayed two absorption bands is their marked acidic character. This dual-band absorption was found when the dye was dissolved in alcohols, which 
are polar, proton donor solvents, with the exception of DMSO and acetonitrile. In contrast, the dye dissolved in aprotic solvents exhibited single-band absorption. Moreover, it is remarkable that the highest quantum yield values corresponded to the dye dissolved in protic solvents, in which the dye displayed two absorption bands. As representative examples of the aforementioned features, Figure 1 shows the normalized absorption and fluorescence emission of 2-Me-4-OMe-TM in acetone, acetonitrile and butanol, representing solvents in which the dye showed either one- or two-band spectral features.
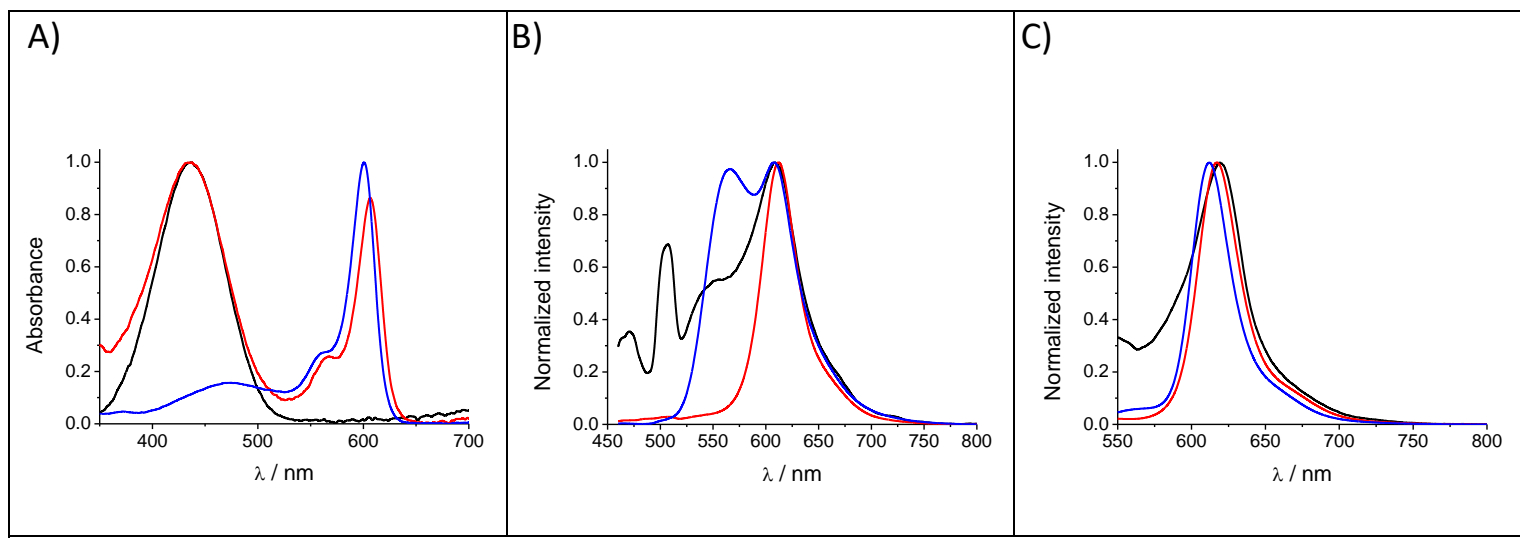

Figure 1. Representative normalized (A) absorption spectra and ( $B$ and $C$ ) emission spectra, with (B) $\lambda_{\mathrm{ex}}=440$ and (C) $\lambda_{\mathrm{ex}}=530 \mathrm{~nm}$, of compound 2-Me-4-OMe-TM in three solvents: acetone (black), acetonitrile (red) and butanol (blue).

Because on its lipophilic character, we have considered the possibility that 2-Me-4-OMe-TM may undergo aggregation in polar solvents, being these aggregates responsible of the redband fluorescence emission. To unravel this, we measured the fluorescence intensity from concentrations as low as $1 \times 10^{-7} \mathrm{M}$, up to high $2-\mathrm{Me}-4-\mathrm{OMe}-\mathrm{TM}$ concentrations $\left(1 \times 10^{-5} \mathrm{M}\right)$ in water (Figure A4A). As aggregation depends on the concentration, low quantities of 2-Me-4OMe-TM should reduce aggregation. To unambiguously discard any aggregation, we plotted the emission intensity vs the 2-Me-4-OMe-TM concentration. Our results showed always the same emission shape in all the concentrations studied, except at the highest concentrations in which auto-absorption caused a slight red-shift in the emission band (see Figure A4B). 
However, selecting another wavelength with less inner filter effect $(620 \mathrm{~nm})$, we obtained a perfect linear behavior in the complete range studied. The fulfillment of the Kavanagh's Law suggests that aggregation in polar solvents can be considered negligible.

To study the solvent dependency based on solvent-dye nonspeficic interactions, we followed the Lippert-Mataga equation (See Appendix A). ${ }^{[17]}$ This equation describes the Stokes shift $(\Delta \underline{v})$ as a consequence of the changes in the dipole moment after the excitation and the dipole energy in solvents with different dielectric constants $(\varepsilon)$ and/or refractive index $(n)$ (Table A3), where both parameters are included in the orientation polarizability $(\Delta f)$.

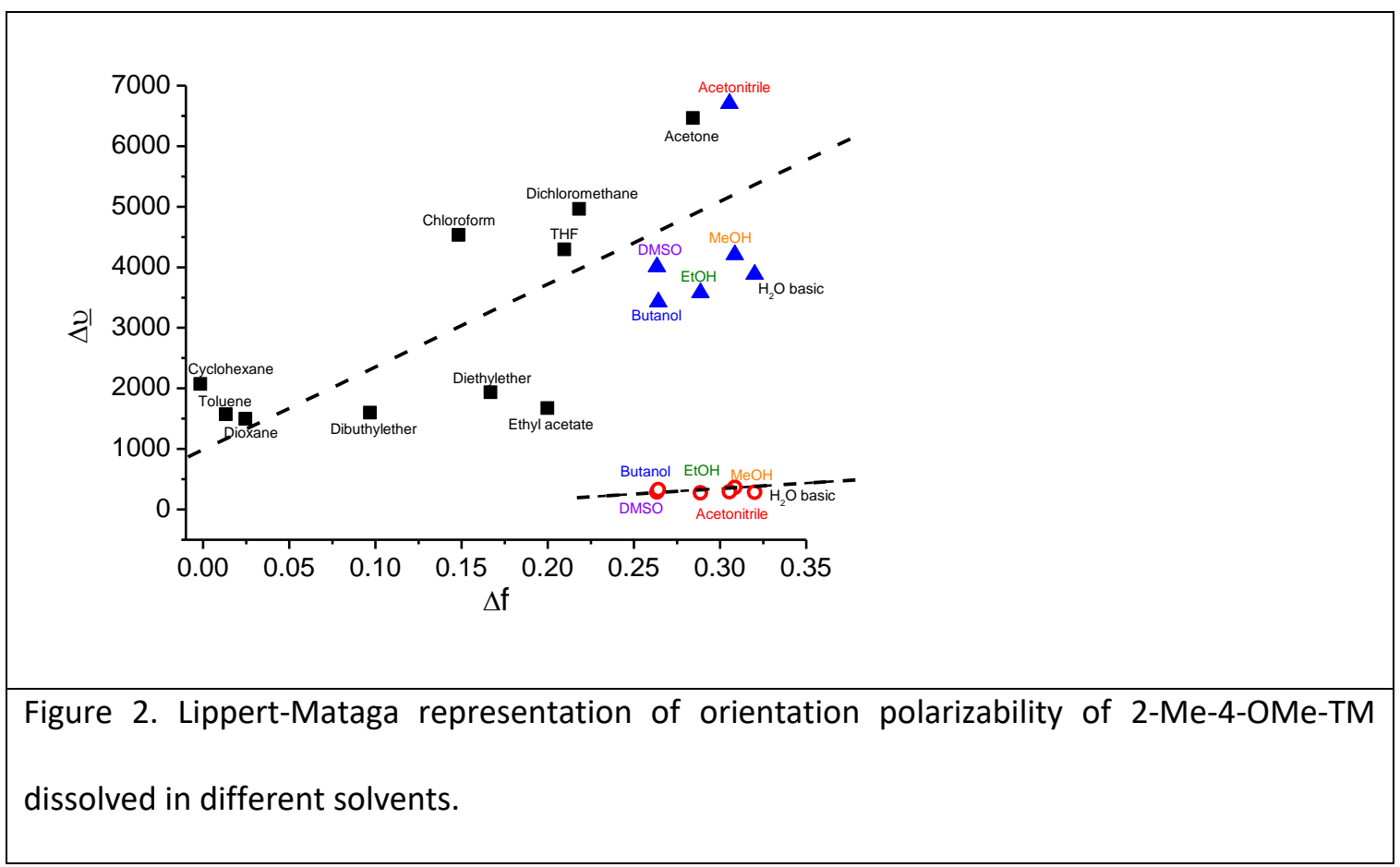

Figure 2 shows the plot of the experimental Stokes shift values versus $\Delta f$, following the Lippert-Mataga representation. For solvents in which the dye presented a double absorption band, we calculated two values: one selecting the blue-shifted (triangles) and the other selecting the red-shifted (open circles) absorption band. We observed two main trends marked by dashed lines. The first trend is observed in those solvents for which the dye presented a 
single band (aprotic solvents) and for the blue-shifted band of the solvents in the protic group. The second trend involves the red-shifted band of the protic solvents. The difference in the trends between aprotic and protic solvents suggests that 2-Me-4-OMe-TM presents specific interactions with solvents such as butanol, ethanol, methanol, acetonitrile or water. ${ }^{[18]}$ This interaction could be the formation of hydrogen bonds between the fluorophore and the solvent; in fact, with the exceptions of acetonitrile and DMSO, the rest of the solvents in this group stand out for their high acidity and proton donor capacity. ${ }^{[19]}$ This fact supports the idea of different electronic densities of the 2-Me-4-OMe-TM dye depending on whether it is forming hydrogen bonds.

The presence of specific hydrogen bonding effects of the solvents makes the Lippert-Mataga formalism insufficient; therefore, we employed a complementary solvatochromic study based on the methodology reported by Catalan. ${ }^{[15]}$ In brief, the analysis is based on four empirical solvent scales, i.e., polarizability (SP), dipolarity (SdP), acidity (SA) and basicity (SB), hence taking into account both general effects and specific hydrogen bonding features of the solvents. The major advantage of the Catalan methodology is that it allows the separation of the relative contribution of the four parameters in the solvatochromic shifts of $\tilde{v}_{a b s}$ and and $\tilde{v}_{e m}$, and hence, it provides insights on which of the solvent's features is the main one responsible for the spectroscopic behavior of the dye. Arithmetically, the effect of the solvent on the physicochemical properties observable $(A)$ is expressed by a multilinear expression:

$$
A=A_{0}+b S A+c S B+d S P+e S d P
$$

where $A$ is a solvent-dependent physicochemical property in a specific solvent, $A_{0}$ is the statistical quantity corresponding to the value of the property in the gas phase and $b$ to $e$ are the regression coefficients describing the sensitivity of property $A$ to the different solutesolvent interactions. ${ }^{[15]} \mathrm{As}$ found in the Lippert-Mataga representation, and due to the dual absorption bands found in protic solvents, we separated them into two different groups, 
depending on the number of absorption bands. In the first dataset, all solvents were considered, using the blue-shifted absorption band for the solvents in which the dye displayed two bands. The second dataset was gathered with the red-shifted absorption band of the dye. We used the correlation coefficient $r$ as the goodness-of-fit criterion, obtaining values of 0.954 and 0.996 for the $A=\tilde{v}_{a b s}$ correlation in the first and the second datasets, respectively. When correlating $A=\tilde{v}_{e m}$, we obtained 0.894 and 0.982 for the two datasets, respectively. These $r$ values support a good correlation in all cases. To understand the importance of each of the solvent's properties, Catalan fitting was carried out, omitting one, two and three solvent scales.

Table A4 shows the correlation coefficient values obtained for all the performed fittings, including all parameters and omitting one of the solvent scales. For the first group, the changes in $\tilde{v}_{a b s}$ were predominantly produced by the solvent acidity and, to a lower extent, basicity, as evidenced by the large negative values and low associated error. This result means that the acid-base features of the solvents are primarily controlling the energy of the transition. This analysis explains the presence of the double band in the acidic solvents (water, methanol, ethanol and butanol) and in those solvents with a combination of low acidity but large basicity (DMSO and acetonitrile).

In contrast, the $\tilde{v}_{e m}$ was largely affected by the solvent's polarizability; hence, the general solvent stabilization of the excited-state is the major effect for the emission, so further stabilization in more dipolar solvents was observed. This result is in excellent agreement with the Lippert-Mataga approach when considering the Stokes shift. Interestingly, when considering the second, red-shifted band, the acidity of the solvent was found to be the major contributor in both $\tilde{v}_{a b s}$ and $\tilde{v}_{e m}$, without a strong dependence on any other physicochemical parameter. 
Therefore, using the Catalan approach, we conclude the following: although acidity is the main physicochemical parameter that affects all solvents and bands for absorption, both groups are affected by different physicochemical parameters for emission. Regarding the first band, the solvent dipolarity represents the primary physicochemical parameter that produces a red-shift in the fluorescence emission. In contrast, our results clearly support the idea of the second absorption and emission band arising from hydrogen bonding interactions with the solvent. Despite the difficulty in quantifying the solvatofluorochromic effects, due to the appearance of specific interactions, this behavior could be considered interesting; the strong differences among solvents due to these specific effects could allow the probe be applied to aqueous or nonaqueous phases, ${ }^{[17]}$ e.g., when the fluorophore enters disperse hydrophobic systems or interacts with a macromolecule.

In addition, we performed the $\left(\mathrm{E}_{\mathrm{T}} 30\right)$ solvent scale polarity ${ }^{[14 \mathrm{~b}, 20]}$ analysis of the absorption and emission maxima (see Figure A5). The correlation of the emission maxima showed two different tendencies regarding the single and blue-shifted band and the red-shifted band. The first group showed a negative solvatofluorochromic behavior whereas the red shifted group exhibited a positive solvatofluorochromic behavior. These interesting differences could indicate the nature of the transition between ground- and excited- level, so that the first group is due to a $\pi \rightarrow \pi^{*}$ transition, whereas the red-shifted band corresponds to a $n \rightarrow \pi^{*}$ transition.

For a better understanding of the transition of the 2-Me-4-OMe-TM fluorescence properties from a polar to a nonpolar medium, we carried out absorption, steady-state and time-resolved fluorescence measurements of 2-Me-4-OMe-TM in different water:THF volume proportions. Figure 3 shows the transition of the absorption and normalized fluorescence intensity by absorption at two different excitation wavelengths (440 and $595 \mathrm{~nm}$ ). The absorption spectra (Figure 3A) showed an evident red-shift of the $~ 570-n m$ absorption band and an increased intensity of the $\sim 440-\mathrm{nm}$ band when the THF proportion was increased. The red band 
completely disappeared in pure THF. The emission spectra also showed striking results. When exciting predominately at the nonpolar maximum (Figure $3 \mathrm{~B} ; \lambda_{\mathrm{ex}}=440 \mathrm{~nm}$ ) and with increasing THF concentrations above $40 \%$, the fluorescence showed a decrease in intensity along with a slight red-shift at approximately $600 \mathrm{~nm}$ and the appearance of an emission shoulder at approximately $550 \mathrm{~nm}$. However, in pure THF, the 2-Me-4-OMe-TM fluorescence decreased strongly, as expected, given the very low quantum yield in THF (0.005). For a better visualization of the two-bands in the fluorescence, we have normalized these spectra (see Figure A6A) where the fluorescence splitting is unambiguously identified when THF is in the media.

Figure $3 \mathrm{C}$ shows the fluorescence emission using the absorption maximum as the excitation wavelength. As can be observed, there was an increase in intensity when the THF proportion was increased to $80 \%$. Interestingly, a red-shift of the emission band was also observed, from $598 \mathrm{~nm}$ in water to $614 \mathrm{~nm}$ in $80 \%$ THF.
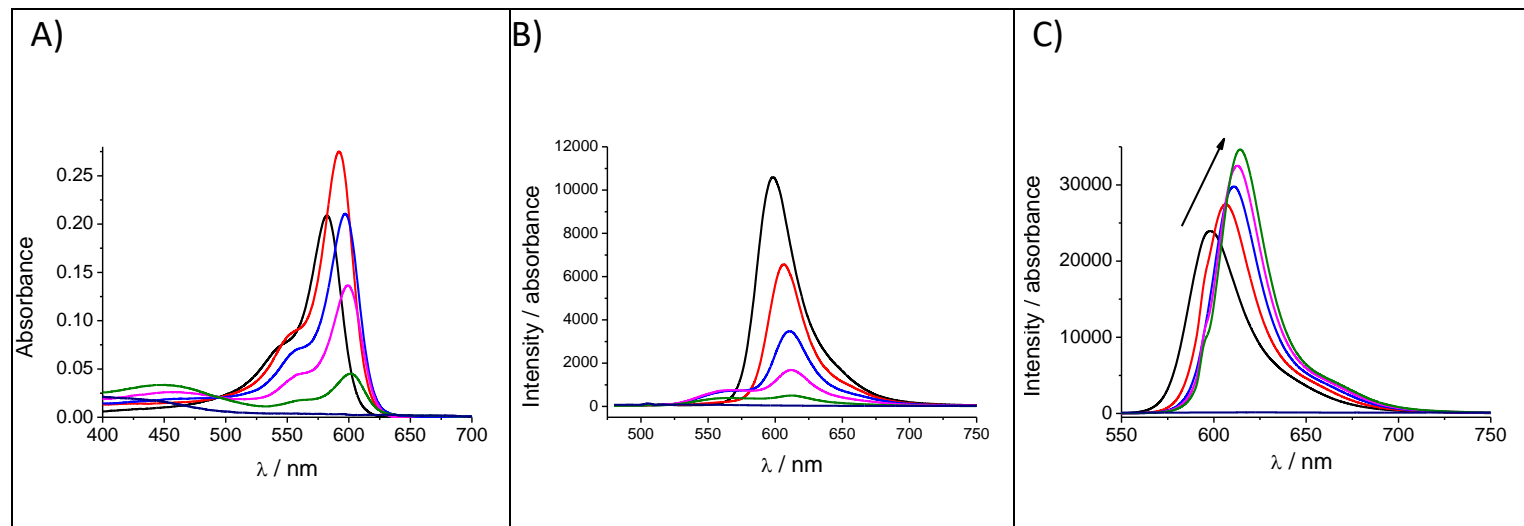

Figure 3. (A) Absorption and (B, C) emission spectra using an excitation wavelength of (B) 440 $\mathrm{nm}$ and (C) $595 \mathrm{~nm}$ in THF:water mixtures with THF proportion of 0 (black), 20 (red), 40 (blue), 60 (pink), 80 (green) and 100\% (dark blue).

We also performed a time-resolved fluorescence study of the dye dissolved in water:THF mixtures using either $530 \mathrm{~nm}$ (Figure 4) or $440 \mathrm{~nm}$ excitation (Figure A6). When 2-Me-4-OMe- 
TM was dissolved in water, the dye presented a single fluorescence lifetime of approximately 3 ns. However, when the THF proportion was increased, the long decay time increased to approximately $4.2 \mathrm{~ns}$, and a second fluorescence lifetime appeared (of approximately $0.2 \mathrm{~ns}$ ) with an increasing relative abundance. Similar trends were observed in both the emission wavelengths of 570 and $600 \mathrm{~nm}$, but with a higher extent at $570 \mathrm{~nm}$ (see Figures 4 and A6). The appearance of the second, and even a third decay time (of approximately $1 \mathrm{~ns}$ ) was even more noticeable using an excitation wavelength of $440 \mathrm{~nm}$ (FigureA6). Using $530 \mathrm{~nm}$, we predominantly excited the 2-Me-4-OMe-TM species, forming hydrogen bonds in protic solvent (i.e., water, with $\tau=3 \mathrm{~ns}$, see Figure $4 \mathrm{a}$ ). However, with the addition of THF, the contribution of this species decreased, favoring the species interacting with the aprotic solvent (see Figure 3a, where the intensity of the red-shifted peak decreased in favor of the blue-shifted peak); showing a decrease in the relative abundance associated with the 3 ns fluorescence lifetime; and increasing the relative abundance associated with the $0.2 / 1$ ns fluorescence lifetimes (see Figure $4 a$ and $4 b$, dashed lines). Because when the excitation wavelength of $530 \mathrm{~nm}$ was used, the 1-ns lifetime was not detected (Figure 4), we can assign this decay time to the probe interacting with the aprotic solvent, whereas the fast decay time of approximately 0.2 ns can be assigned to dynamics of the interconversion and formation of the hydrogen bonding species.

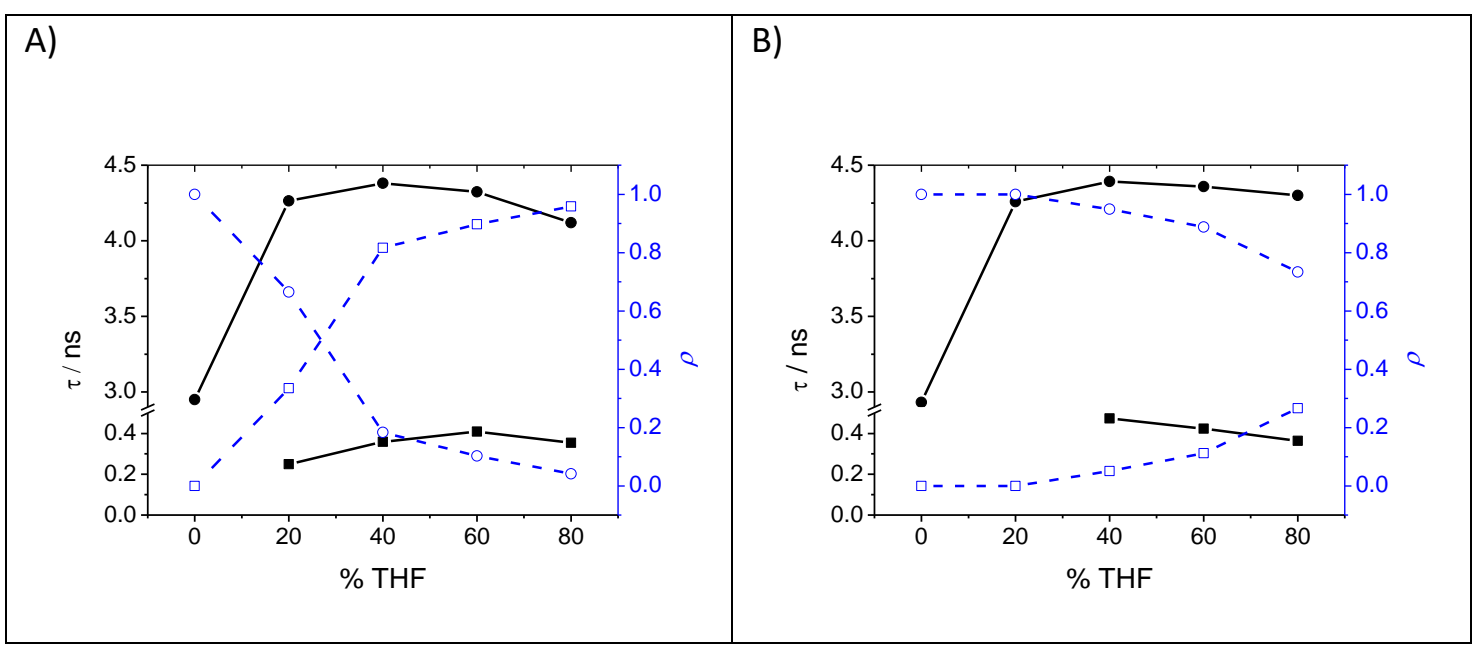


Figure 4. Fluorescence lifetime $(\tau)$ and relative abundance $(\rho)$ at $\lambda_{\text {ex }}=530 \mathrm{~nm}$ at A) $\lambda_{\text {em }}=570$ and B) $600 \mathrm{~nm}$.

Considering the increase in the fluorescence lifetime during the transition from protic to aprotic solvent, we deliberated its applicability to detect polarity changes in the medium using the fluorescence lifetime as the analytical signal, hence making the approach suitable for fluorescence lifetime imaging microscopy (FLIM), as a polarity probe in cells. For this purpose, we suggest its use by selecting the best conditions where the sample maintains a monoexponential decay nature and with higher relative abundance (i.e., $\lambda_{\mathrm{ex}}=530 \mathrm{~nm}$ and $\lambda_{\mathrm{em}}$ $=600 \mathrm{~nm}$ ). For a more complete study of the changes in its properties and its behavior in the different compartments of the cells, we previously studied the photophysics of 2-Me-4-OMeTM in a micellar system.

\subsection{Study of 2-Me-4-OMe-TM excited-state dynamics in water and SDS micelles}

Once the solvatofluorochromic behavior of 2-Me-4-OMe-TM was established, we investigated whether the interaction with the cellular membranes was the reason behind the decreased sensitivity towards phosphate. For this assessment, we compared the phosphate-mediated ESPT reaction of the dye in water and in SDS micelles.

As we showed with green-emitting xanthene dyes, ${ }^{[6 a]}$ the presence of a phosphate-mediated ESPT reaction served as the basis for their use as intracellular phosphate sensors. Through a fluorogenic mechanism, we previously showed the application of a modified 2-Me-4-OMe-TM as a dual sensor for the simultaneous detection of intracellular phosphate ions and biothiols. $^{[12]}$ Since a full study of the ESPT reaction for the dye 2-Me-4-OMe-TM was lacking, we investigated its acid-base equilibrium around physiological pH by means of absorption, steady-state and time-resolved fluorescence spectroscopy (See Appendix A, Figures A7-A13). More interestingly, all the excited-state rate constants defining the excited-state process were 
calculated (Scheme A2, FigureA13 and Table A5). The presence of the ions of the phosphate buffer at near-neutral pH effectively promotes the ESPT reaction, causing a phosphatedependent decrease in the fluorescence lifetime of the dye (FigureA13B). The solution of the excited-state kinetic scheme in the presence of phosphate would allow the use of 2-Me-4OMe-TM as a FLIM sensor for phosphate in biological samples. However, when this dye was used intracellularly, the sensitivity was lower than expected, and specific calibration procedures were employed. ${ }^{[12]}$ Therefore, as cytoplasm is a complex matrix with multiple compartments and microenvironments, we decided to evaluate the effects of membranous systems on the photophysical properties of 2-Me-4-OMe-TM. A simpler approach to studying the complex intracellular system is the use of micellar medium.

We carried out a study of the behavior of 2-Me-4-OMe-TM in SDS micelles at different phosphate concentrations and $\mathrm{pH} 7.35$. Figure 5 shows the absorption and emission spectra at $1 \mathrm{mM}$ SDS concentration and at different phosphate concentrations. Figures A14 and A15 show the absorption and emission spectra at different SDS concentrations. The addition of phosphate produced notable changes in the absorption spectra, causing a decrease in the 595 $\mathrm{nm}$ maximum and an increase at approximately $460 \mathrm{~nm}$. This change may be due to the interaction of the dye with the micelles that formed in solution. This interaction may produce a change in the polarity of the surroundings of the dye, favoring aprotic interactions of the probe inside the micelles. ${ }^{[21]}$ Following the fluorescence intensity, we calculated the critical micelle concentration (CMC) of the SDS (see Figure A16) in the presence of different concentrations of phosphate buffer. We found a decrease in the CMC from approximately 2.05 $\mathrm{mM}$ to $0.95 \mathrm{mM}$, indicating that the phosphate concentration favored micellar formation. This result can be attributed to the effect of the electrolyte on the micelle formation, which reduced the thickness of the ionic layer surrounding the polar moiety of the surfactant and allowed micellar formation via electrostatic forces. ${ }^{[22]}$ 


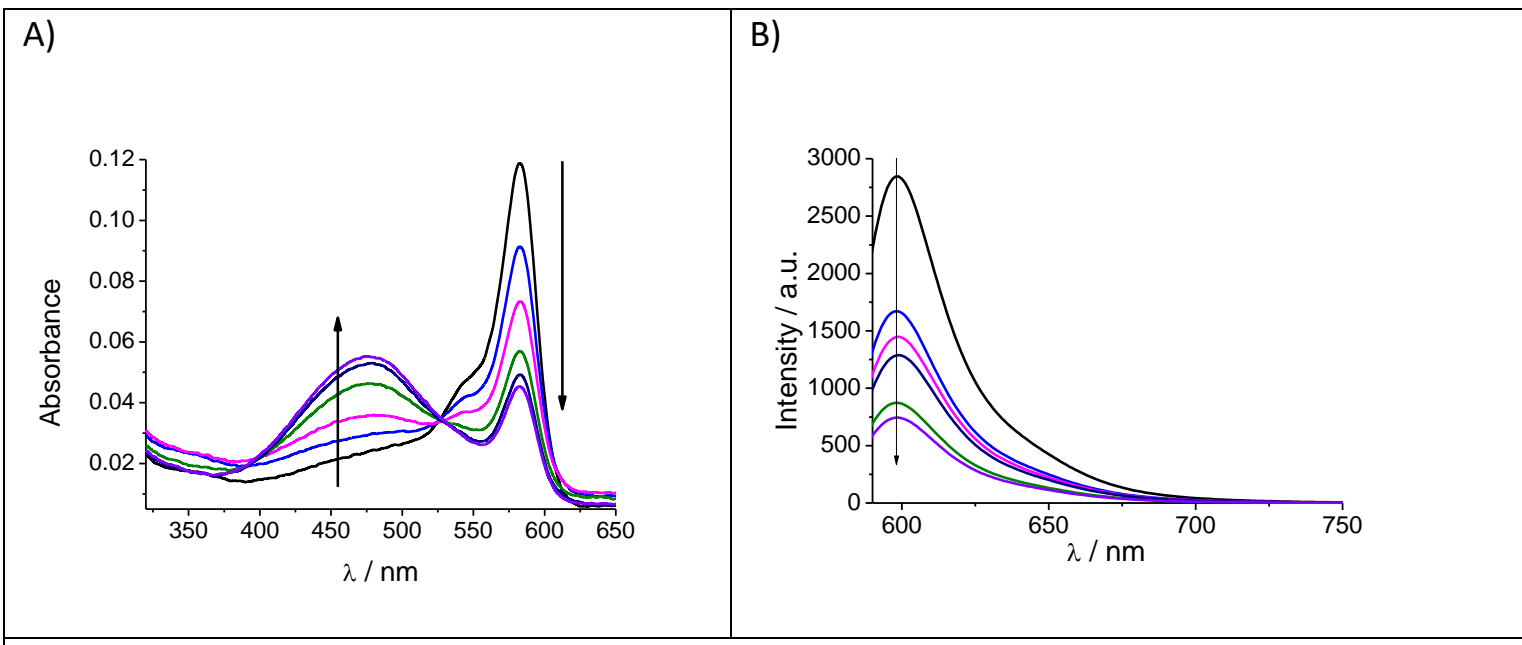

Figure 5. A) Absorption and B) Emission spectra $\left(\lambda_{\text {ex }}=583 \mathrm{~nm}\right)$ of 2-Me-4-OMe-TM at $1 \mathrm{mM}$ of SDS and different phosphate concentration $(5,50,100,150,175$ and $200 \mathrm{mM})$ at pH 7.35. Arrows indicate the change in the spectra due to the increase in the phosphate concentration.

Next, we measured the time-resolved fluorescence of 2-Me-4-OMe-TM at different SDS and phosphate concentrations (Figure 6A). We observed two different behaviors: when the SDS concentration was below the $\mathrm{CMC}$, between 0.1 and $1 \mathrm{mM}$, the dye's lifetime decreased with the addition of phosphate, in agreement with the buffer-mediated ESPT reaction. However, when the SDS concentration was above the $\mathrm{CMC}$, between 3 and $20 \mathrm{mM}$, the lifetime of the dye increased due to the change in the polarity of the dye's surroundings and phosphate acting as a promoter of micelle formation.

Therefore, below the CMC, the effect of the phosphate buffer-mediated ESPT reaction prevailed, producing a decrease in the fluorescence lifetime. ${ }^{[12]}$ In contrast, above the $\mathrm{CMC}$, the phosphate buffer acted as a stabilizer of the micellar medium, and as a consequence, more micelles were formed. This process decreased the concentration of the anionic species of the dye but, at the same time, resulted in a solvent protective effect that caused an increase in the fluorescence lifetime. 


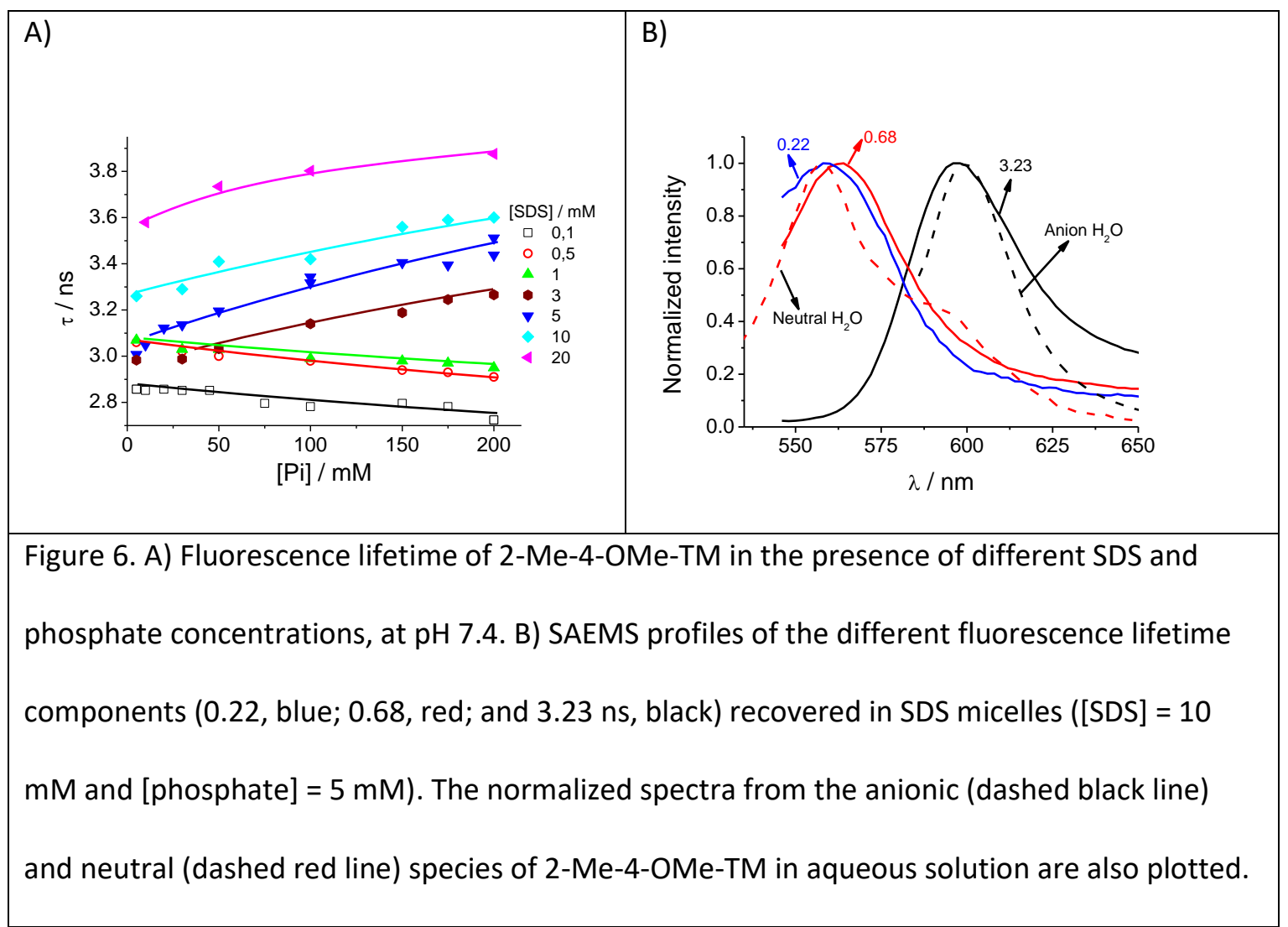

Finally, to identify each of the species in the micellar system unambiguously, through timeresolved data, we calculated the species-associated emission spectra (SAEMS) of each of the decay time components. Figure $6 B$ shows the SAEMS obtained from the three fluorescence lifetimes obtained in the micellar medium $([S D S]=10 \mathrm{mM}$ and [phosphate] $=5 \mathrm{mM}$ ). Moreover, in Figure 6B, the emission spectra of the two prototropic species involved in aqueous solution (dashed lines) are also presented for comparison. The species with $\tau=3.23$ ns presented a maximum at $600 \mathrm{~nm}$, which clearly overlapped with the anionic species in aqueous solution at $\mathrm{pH}$ 9.00. Therefore, this spectrum can be associated with the anionic species of the 2-Me-4-OMe-TM. We assign the slight red-shift to the difference in the polarity between the aqueous and SDS solutions. We used the same argument to identify the neutral species of the fluorophore, which prevails in aqueous solution at $\mathrm{pH} 4.00$, overlapping with the SAEMS of the component with $\tau=0.22 \mathrm{~ns}$. The third component, with $\tau=0.68 \mathrm{~ns}$, presented a 
SAEMS with a maximum that was red-shifted with respect to the neutral species. The presence of this fluorescence lifetime can be associated with the interaction of the dye and the micelles, which could be produced in the interior of the micelles or in the interphase. A similar behavior was found in other xanthene derivatives when introduced in reverse micelles ${ }^{[23]}$ or interacting with macromolecules. ${ }^{[24]}$

Anionic species are most stabilized in the excited state by water molecules or other protic solvents, producing a decrease in the difference of energy between the ground and excited states. This stabilization is not present in aprotic solvents, as in micelles, avoiding this stabilization and producing a blue-shifted effect. ${ }^{[25]}$ Both species are preferentially located in their microenvironment, so the longest fluorescence lifetime is associated with the anionic form that remains in the solvent despite the micelles. Although the quantity of anionic species is decreased, its fluorescence lifetime is dependent on the polarity of the solvent, which is determined by the number of micelles formed. We consider the micellar system as an approximation to simulate the cellular interior. The studied fluorophore could be used as a polarity sensor for different intracellular compartments.

\subsection{Bioimaging}

First, we checked the intracellular uptake and accumulation of 2-Me-4-OMe-TM in HEK293 cells. The fluorescence intensity images are presented in Figures 7A-C (and Figure A17), representing a time point 30 seconds after the fluorophore addition to the extracellular medium. These intensity images show a specific accumulation pattern similar to previous silicon-substituted xanthenes. ${ }^{[5]}$ Moreover, the corresponding lifetime images (Figures 7D-F, and Figure A17) exhibited differences in the average lifetime values, which were dependent on the microenvironment polarity of the regions where the probe was located. 


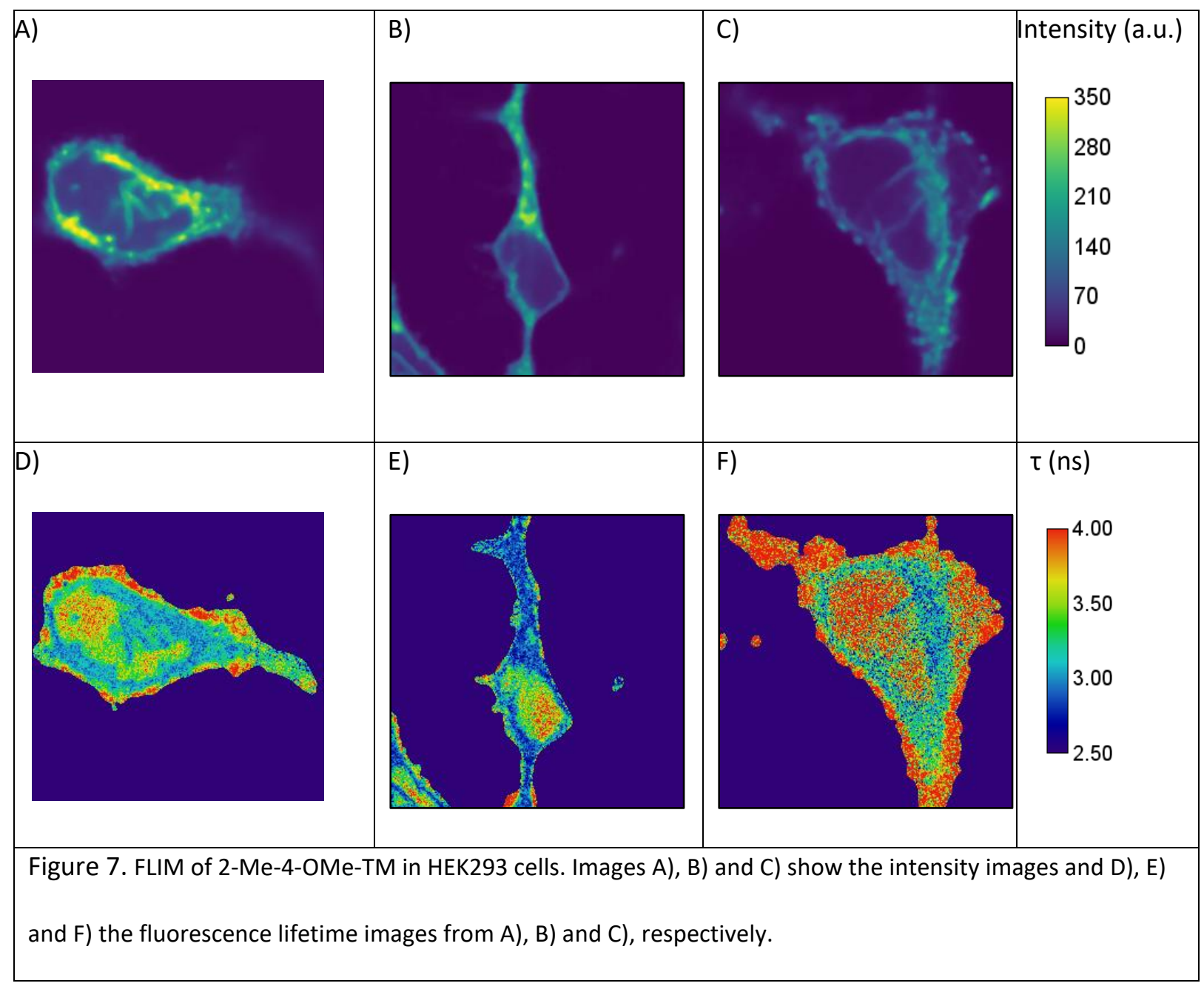

As observed in the FLIM images, there are three well-differentiated lifetimes in the cells: the structure surrounding the cell, the nuclei and the cytoplasmic regions. The three average fluorescence lifetimes recovered from these three regions of a total of seven cells were $3.96 \pm$ $0.08,3.67 \pm 0.09$ and $3.00 \pm 0.09 \mathrm{~ns}$, respectively. The anionic form was predominantly excited in these FLIM images, and it presented a longer lifetime in a nonpolar environment, such as the surrounding structures that can be associated with the plasma membranes and other structures associated with proteins and other macromolecules. The dye located at the nuclei also presented longer fluorescence lifetime values than that dissolved in the cytoplasm but lower than that incorporated in the plasma membrane structures. This result could be explained by the less polar microenvironment, typical of the interior of the nucleus. ${ }^{[26]}$ Finally, 
the lowest fluorescence lifetime of the dye was found in the cytoplasm, which presented the highest polarity.

However, while we analyzed the cytoplasmic region as a whole, a more detailed inspection of the intensity images allowed the clear discrimination of different structures that could be associated with mitochondria and other intracellular organelles (see Figure 8 and Figure A18). Although the mechanism of the incorporation of the dye into these structures is not well known, there are some hypotheses that imply the chemical equilibrium between the neutral and anionic forms and the accumulation of the anion formic inside the mitochondria due to the slightly higher intramitochondrial $\mathrm{pH}^{[27]}$ According to these authors, only the more lipophilic neutral form is able to cross the mitochondrial membrane; inside mitochondria, the higher $\mathrm{pH}$ shifts the chemical equilibrium to favor the anionic form; and as this species is less lipophilic, it is retained inside the organelle. In any case, the accumulation of the probe in these structures allows easy discrimination of the regions with lower intensity and therefore isolation to study the polarity of these structures. Figures 8A-D and A18 show the complete FLIM images and the isolated regions using an intensity mask (where only the more intense pixels were selected), which correspond to different intracellular structures. Interestingly, the analyzed region of interest (Figure $9 C-D$ and $A 18$ ) presented two different fluorescence lifetimes, indicating different environments. 


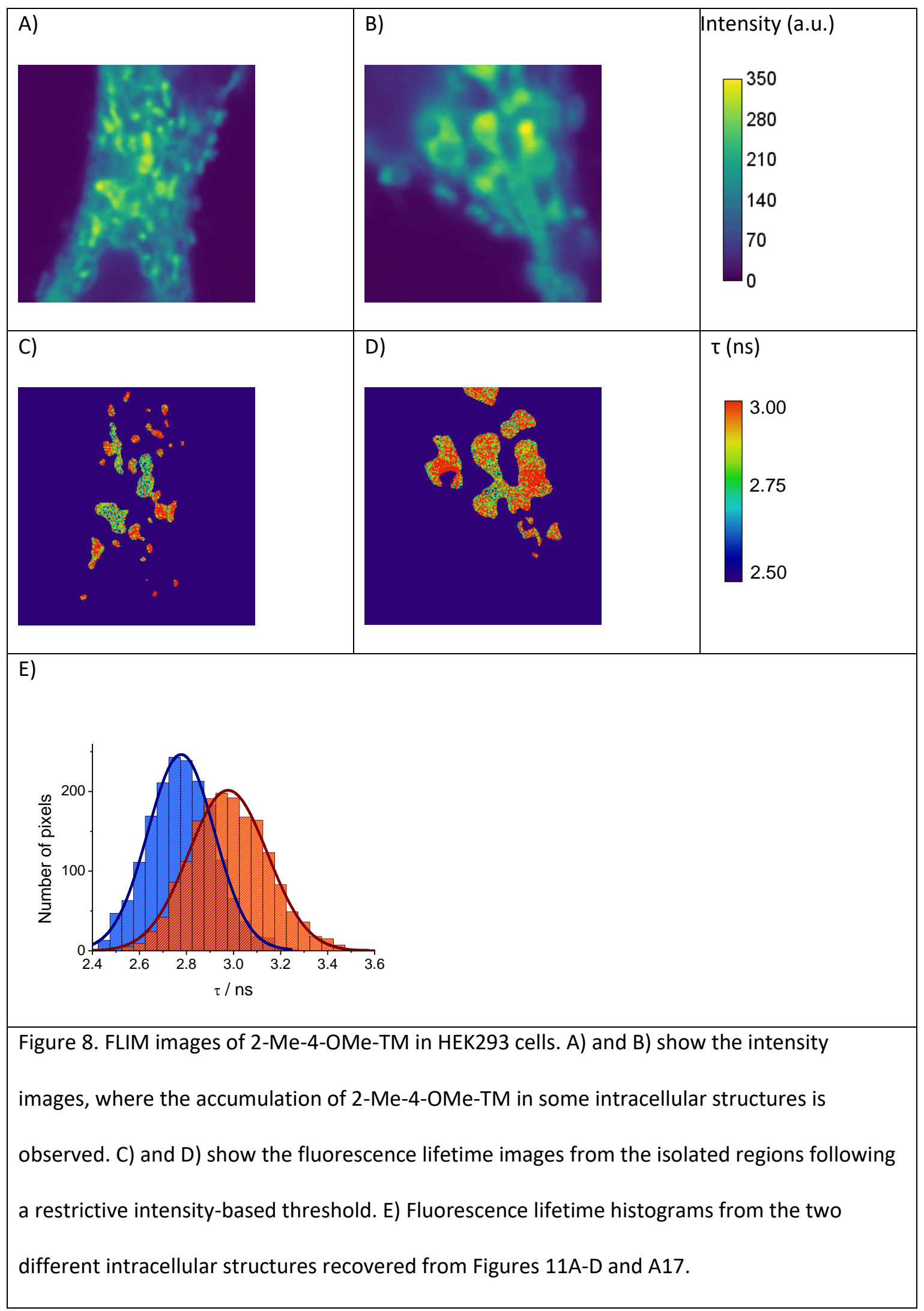


We calculated the histograms of fluorescence lifetimes coming from these two intracellular structures. The distributions are shown in Figure 9E, where two clearly different Gaussian curves were obtained. This probe accumulation in intracellular organelles together with the dye's capacity to measure polarity via the fluorescence lifetime could be useful in cancerrelated biomedical research since polarity changes in tumoral mitochondria have been described. ${ }^{[28]}$

\section{Conclusions}

In this work, we performed a thorough photophysical study of the probe 2-Me-4-OMe-TM. From this study, we found that the dye is suitable for detecting phosphate through a buffermediated ESPT reaction in aqueous solution. Moreover, it has been demonstrated that 2-Me4-OMe-TM presents high sensitivity to the solvent's acidity, forming specific hydrogen bonding interactions, and polarity. The polarity affects the equilibrium of the prototropic species but also increases the fluorescence lifetime of the anionic form. The kosmotropic effect of the phosphate buffer on micelle formation was observed through the fluorescence emission of 2Me-4-OMe-TM. Finally, we used the probe to detect differences in polarity between different regions of cells using FLIM microscopy. We found a specific pattern of intensity where the probe accumulates in some organelles. This finding allowed the isolation of these organelles to determine their polarity.

\section{Acknowledgements}

This work was funded by grants CTQ2017-85658-R, CTQ2014-55474-C2-2-R (Spanish Ministry of Economy and Competitiveness; Agencia Estatal de Investigacion, AEl; and European Regional Development Fund, ERDF), QM2012-790 (Junta de Andalucía), and a grant from the Fundación Botín.

\section{$\underline{\text { References }}$}


[1] a) T. Ueno, Y. Urano, K. Setsukinai, H. Takakusa, H. Kojima, K. Kikuchi, K. Ohkubo, S. Fukuzumi, T. Nagano, Journal of the American Chemical Society 2004, 126, 1407914085; b) L. F. Mottram, S. Boonyarattanakalin, R. E. Kovel, B. R. Peterson, Organic Letters 2006, 8, 581-584; c) A. Martinez-Peragon, D. Miguel, R. Jurado, J. Justicia, J. M. Alvarez-Pez, J. M. Cuerva, L. Crovetto, Chemistry 2014, 20, 447-455.

[2] a) T. Egawa, Y. Koide, K. Hanaoka, T. Komatsu, T. Terai, T. Nagano, Chemical Communications 2011, 47, 4162-4164; b) J. B. Grimm, T. A. Brown, A. N. Tkachuk, L. D. Lavis, ACS Central Science 2017, 3, 975-985.

[3] a) T. Ikeno, T. Nagano, K. Hanaoka, Chemistry - An Asian Journal 2017, 12, 1435-1446; b) F. Deng, Z. Xu, Chinese Chemical Letters 2018, in press DOI: https://doi.org/10.1016/j.cclet.2018.12.012.

[4] a) Y. Kushida, T. Nagano, K. Hanaoka, Analyst 2015, 140, 685-695; b) K. Hirabayshi, K. Hanaoka, T. Takayanagi, Y. Toki, T. Egawa, M. Kamiya, T. Komatsu, T. Ueno, T. Terai, K. Yoshida, M. Uchiyama, T. Nagano, Y. Urano, Analytical Chemistry 2015, 87, 9061-9069.

[5] L. Crovetto, A. Orte, J. M. Paredes, S. Resa, J. Valverde, F. Castello, D. Miguel, J. M. Cuerva, E. M. Talavera, J. M. Alvarez-Pez, Journal of Physical Chemistry A 2015, 119, 10854-10862.

[6] a) J. M. Paredes, M. D. Giron, M. J. Ruedas-Rama, A. Orte, L. Crovetto, E. M. Talavera, R. Salto, J. M. Alvarez-Pez, Journal of Physical Chemistry B 2013, 117, 8143-8149; b) Q. Zhang, K. Xiao, J. M. Paredes, T. Mamonova, W. B. Sneddon, H. Liu, D. Wang, S. Li, J. C. McGarvey, D. Uehling, R. Al-awar, B. Joseph, F. Jean-Alphonse, A. Orte, P. A. Friedman, Journal of Biological Chemistry 2019.

[7] M. J. Ruedas-Rama, J. M. Alvarez-Pez, L. Crovetto, J. M. Paredes, A. Orte, in Advanced Photon Counting: Applications, Methods, Instrumentation (Eds.: P. Kapusta, M. Wahl,

R. Erdmann), Springer International Publishing, Cham, 2015, pp. 191-223.

[8] C. Hille, M. Berg, L. Bressel, D. Munzke, P. Primus, H. G. Löhmannsröben, C. Dosche, Analytical and Bioanalitical Chemistry 2008, 391, 1871-1879.

[9] K. Sagolla, H. G. Lohmannsroben, C. Hille, Analytical and Bioanalytical Chemistry 2013, $405,8525-8537$.

[10] K. Okabe, N. Inada, C. Gota, Y. Harada, T. Funatsu, S. Uchiyama, Nature Communications 2012, 3.

[11] a) M. K. Kuimova, G. Yahioglu, J. A. Levitt, K. Suhling, Journal of the American Chemical Society 2008, 130, 6672-6673; b) L. E. Shimolina, M. A. Izquierdo, I. Lopez-Duarte, J. A. Bull, M. V. Shirmanova, L. G. Klapshina, E. V. Zagaynova, M. K. Kuimova, Scientific Reports 2017, 7.

[12] P. Herrero-Foncubierta, J. M. Paredes, M. D. Giron, R. Salto, J. M. Cuerva, D. Miguel, A. Orte, Sensors 2018, 18, 18.

[13] a) G. S. Loving, M. Sainlos, B. Imperiali, Trends in Biotechnology 2010, 28, 73-83; b) A. S. Klymchenko, Y. Mely, in Fluorescence-Based Biosensors: From Concepts to Applications, Vol. 113 (Ed.: M. C. Morris), 2013, pp. 35-58.

[14] a) A. Marini, A. Munoz-Losa, A. Biancardi, B. Mennucci, Journal of Physical Chemistry $B$ 2010, 114, 17128-17135; b) C. Reichardt, Chemical Reviews 1994, 94, 2319-2358; c) V. G. Machado, R. I. Stock, C. Reichardt, Chemical Reviews 2014, 114, 10429-10475; d) R. Mera-Adasme, M. Domínguez, Physical Chemistry Chemical Physics 2018, 20, 1812718132.

[15] J. Catalan, Journal of Physical Chemistry B 2009, 113, 5951-5960.

[16] J. Schindelin, I. Arganda-Carreras, E. Frise, V. Kaynig, M. Longair, T. Pietzsch, S. Preibisch, C. Rueden, S. Saalfeld, B. Schmid, J. Y. Tinevez, D. J. White, V. Hartenstein, K. Eliceiri, P. Tomancak, A. Cardona, Nature Methods 2012, 9, 676-682.

[17] J. R. Lakowicz, Principles of Fluorescence Spectroscopy, 3rd ed., Springer, 2006.

[18] D. Patra, C. Barakat, Spectrochimica Acta Part a-Molecular and Biomolecular Spectroscopy 2011, 79, 1034-1041. 
[19] C. J. Talone, J. Y. Gao, J. R. Lynch, R. M. Tanu, S. T. Deyrup, Spectrochimica Acta Part aMolecular and Biomolecular Spectroscopy 2016, 156, 138-142.

[20] J. P. Cerón-Carrasco, D. Jacquemin, C. Laurence, A. Planchat, C. Reichardt, K. Sraïdi, Journal of Physical Organic Chemistry 2014, 27, 512-518.

[21] A. Mallick, S. C. Bera, S. Maiti, N. Chattopadhyay, Biophysical Chemistry 2004, 112, 914.

[22] E. Fuguet, C. Rafols, M. Roses, E. Bosch, Analytica Chimica Acta 2005, 548, 95-100.

[23] A. Orte, M. J. Ruedas-Rama, J. M. Paredes, L. Crovetto, J. M. Alvarez-Pez, Langmuir 2011, 27, 12792-12799.

[24] J. M. Paredes, L. Crovetto, A. Orte, S. G. Lopez, E. M. Talavera, J. M. Alvarez-Pez, The Journal of Physical Chemistry A 2011, 115, 13242-13250.

[25] P. P. Mishra, A. L. Koner, A. Datta, Chemical Physics Letters 2004, 400, 128-132.

[26] F.-X. Theillet, A. Binolfi, T. Frembgen-Kesner, K. Hingorani, M. Sarkar, C. Kyne, C. Li, P. B. Crowley, L. Gierasch, G. J. Pielak, A. H. Elcock, A. Gershenson, P. Selenko, Chemical Reviews 2014, 114, 6661-6714.

[27] X. Q. Xiong, F. L. Song, G. W. Chen, W. Sun, J. Y. Wang, P. Gao, Y. K. Zhang, B. Qiao, W. F. Li, S. G. Sun, J. L. Fan, X. J. Peng, Chemistry-a European Journal 2013, 19, 6538-6545.

[28] H. Zhu, J. Fan, J. Du, X. Peng, Accounts of Chemical Research 2016, 49, 2115-2126. 


\section{APPENDIX A}

\section{A solvatofluorochromic silicon-substituted xanthene dye useful in bioimaging}

Laura Espinar-Barranco ${ }^{a}$, Pilar Luque-Navarro ${ }^{a}$, Maja Ana Strnad ${ }^{a}$, Pilar Herrero-Foncubierta ${ }^{a, c}$, Luis Crovetto ${ }^{a}$, Delia Miguel ${ }^{a}$, Maria D. Giron ${ }^{b}$, Angel Orte ${ }^{a}$, Juan M. Cuervac ${ }^{c}$ Rafael Salto ${ }^{b}$, Jose M. Alvarez-Pez ${ }^{\mathrm{a}}$, Jose M. Paredes ${ }^{\mathrm{a}}$

[a] Departamento de Fisicoquimica. Facultad de Farmacia Unidad de Excelencia en Quimica Aplicada a Biomedicina y Medioambiente (UEQ). Universidad de Granada. Campus Cartuja, 18071 Granada (Spain)

[b] Departamento de Bioquímica y Biología Molecular. Facultad de Farmacia. Unidad de Excelencia en Quimica Aplicada a Biomedicina y Medioambiente (UEQ). Universidad de Granada. Campus Cartuja, 18071 Granada (Spain)

[c] Departamento de Química Orgánica. Facultad de Ciencias. Unidad de Excelencia en Quimica Aplicada a Biomedicina y Medioambiente (UEQ). C. U. Fuentenueva s/n, 18071 Granada (Spain)

\begin{tabular}{|l|l|}
\hline Synthesis & A3 \\
\hline Sample preparation & A3 \\
\hline Scheme A1 & A3 \\
\hline Quantum Yield calculation & A4 \\
\hline Figure A1 & A5 \\
\hline Figure A2 & A5 \\
\hline Figure A3 & A5 \\
\hline Figure A4 & A6 \\
\hline Figure A5 & A6 \\
\hline Table A1 & A7 \\
\hline Table A2 & A8 \\
\hline Table A3 & A9 \\
\hline
\end{tabular}




\begin{tabular}{|c|c|}
\hline Lippert Mataga equation & A10 \\
\hline Table A4 & A11 \\
\hline Figure A6 & A12 \\
\hline Absorption studies & A12 \\
\hline $\begin{array}{l}\text { Spectroscopy and photophysical properties of 2-Me-4-OMe-TM in aqueous } \\
\text { solution and sensitivity towards phosphate ions }\end{array}$ & A13 \\
\hline Scheme A2 & A14 \\
\hline Figure A7 & A16 \\
\hline Figure A8 & A16 \\
\hline Figure A9 & A17 \\
\hline Steady-state fluorescence studies & A17 \\
\hline Figure A10 & A18 \\
\hline Figure A11 & A18 \\
\hline Figure A12 & A19 \\
\hline Buffer-mediated ESPT & A19 \\
\hline Figure A13 & A21 \\
\hline Table A5 & A22 \\
\hline Figure A14 & A23 \\
\hline Figure A15 & A24 \\
\hline Figure A16 & A25 \\
\hline Figure A17 & A26 \\
\hline Figure A18 & A27 \\
\hline
\end{tabular}


Synthesis

2-Me-4-OMe-TM has been synthesized as previously described. ${ }^{[1]}$ In short, synthesis of the compound 2-Me-4-OMe-TM was carried out by nucleophilic addition of the organolithium derivative generated by the reaction of 4-bromo-3-methoxyphenol with $n$-BuLi to the previously described ketone (I, see Scheme A1). ${ }^{[2]}$ Later treatment with diluted $\mathrm{HCl}$ afforded compound 2-Me-4-OMe-TM in a 70\% yield.

The purification of 2-Me-4OMe-TM was carried out by flash chromatography on Merck silica gel 50 using $\mathrm{CH}_{2} \mathrm{Cl}_{2}: \mathrm{MeOH}$ mixtures. A subsequent purification step was performed using preparative TLC in the same mixture of solvents. The determination and characterization of 2Me-4OMe-TM was carried out by ${ }^{1} \mathrm{H}-\mathrm{NMR},{ }^{13} \mathrm{C}-\mathrm{NMR}$ and high resolution mass spectrometry (HRMS)

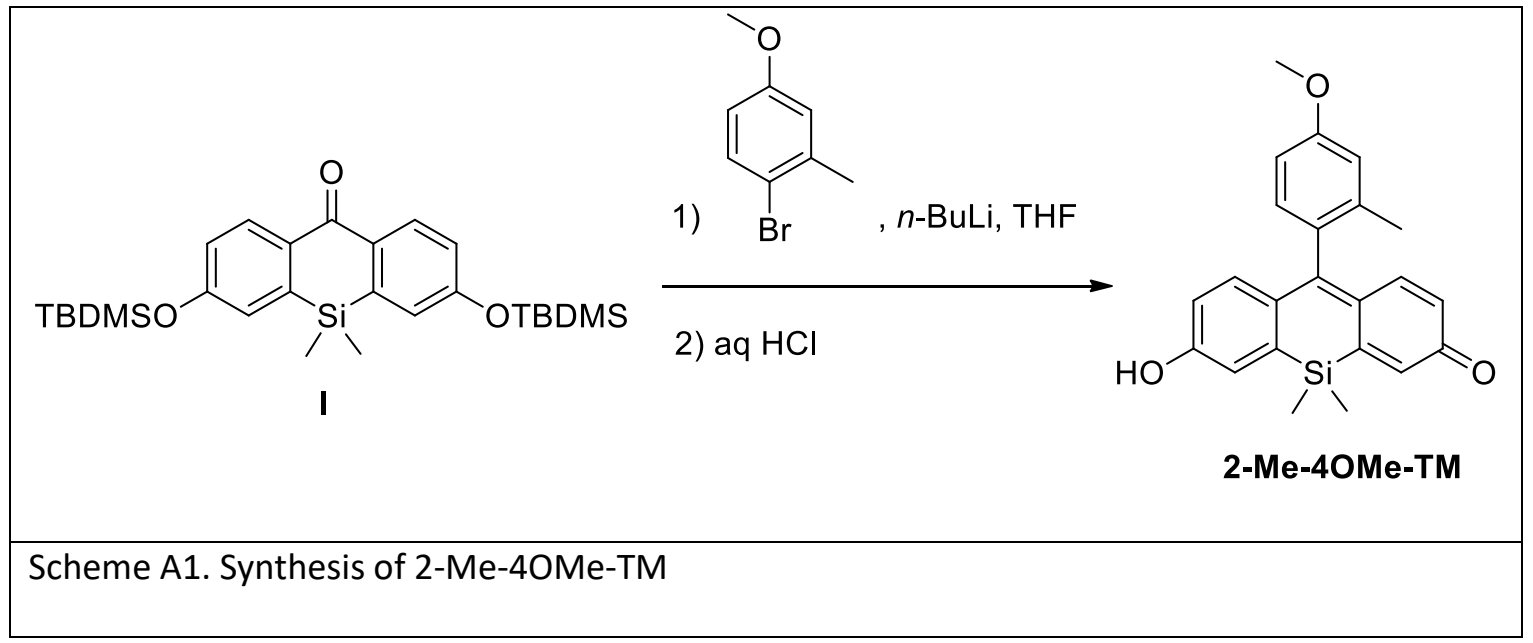

\section{Sample preparation}

A stock solution of 2-Me-4-OMe-TM was prepared by dissolving the purified solid in DMSO at a concentration of $4 \times 10^{-4} \mathrm{M}$. The buffer reagents used were $\mathrm{NaH}_{2} \mathrm{PO}_{4} \cdot \mathrm{H}_{2} \mathrm{O}$ and $\mathrm{Na}_{2} \mathrm{HPO}_{4} \cdot 7 \mathrm{H}_{2} \mathrm{O}$ (Sigma-Aldrich, Spain). Stock solutions (0.5 M) of these reagents were prepared. All the aqueous solutions were prepared with ultrapure water $(18.2 \mathrm{M} \Omega-\mathrm{cm})$. When necessary, the $\mathrm{pH}$ 
was adjusted using $0.1 \mathrm{M} \mathrm{NaOH}$ or $0.1 \mathrm{M} \mathrm{HClO}_{4}$. 1-Butanol, acetone, acetonitrile, chloroform, cyclohexane, dibutylether, diethylether, dioxane, DMSO, ethyl acetate, ethanol, methanol, THF and toluene were all from Sigma-Aldrich. A stock solution of $50 \mathrm{mM} \mathrm{SDS},\left(\mathrm{NaC}_{12} \mathrm{H}_{25} \mathrm{SO}_{4}\right)(\mathrm{Sigma}-$ Aldrich) was prepared. Micelle samples were prepared using different SDS concentrations and adding different phosphate concentrations from the stock solutions. The $\mathrm{pH}$ values were maintained at 7.35 .

\section{$\underline{\text { Quantum Yield calculation }}$}

The relative fluorescence quantum yield values were determined using the following formula ${ }^{[3]}$ :

$$
\Phi=\Phi_{R} \cdot \frac{\mathrm{I}}{I_{R}} \cdot \frac{O D_{R}}{\mathrm{OD}} \cdot \frac{n^{2}}{n_{R}^{2}}
$$

Where $\Phi$ and $\Phi_{R}$ denote the fluorescence quantum yield of the sample and the reference respectively, $I$ and $I_{R}$ the integrated fluorescence spectra of the sample and the reference, OD and $O D_{R}$ the absorption at the excitation wavelength of the sample and the reference and $n$ and $n_{R}$ the refractive index of the solvent where the sample and reference are dissolved. As references, we have used fluorescein in $0.01 \mathrm{M} \mathrm{NaOH}(\Phi=0.91)^{[4]}$ for samples that were excited at $440 \mathrm{~nm}$ with a range of emission corresponding to the blue-shifted peaks, and rhodamine 101 in ethanol $(\Phi=0.98)^{[5]}$ for samples that were excited at $530 \mathrm{~nm}$ and a range of emission corresponding to the red-shifted peaks.

The emission spectra were measured at excitation wavelength of 440 , and $530 \mathrm{~nm}$, using $5 \mathrm{~nm}$ and $2.5 \mathrm{~nm}$ for excitation and emission slits at room temperature. 


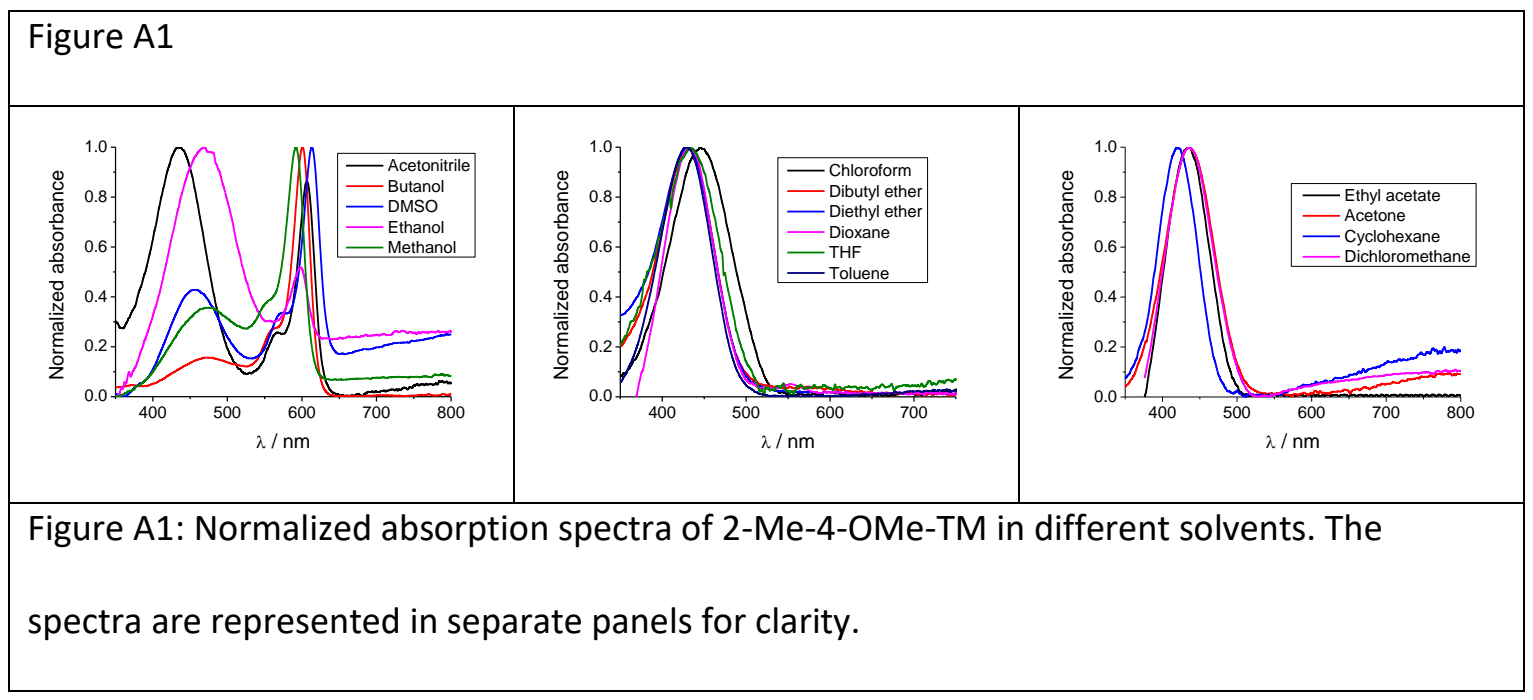

\begin{tabular}{|c|c|c|}
\hline \multicolumn{3}{|l|}{ Figure A2 } \\
\hline 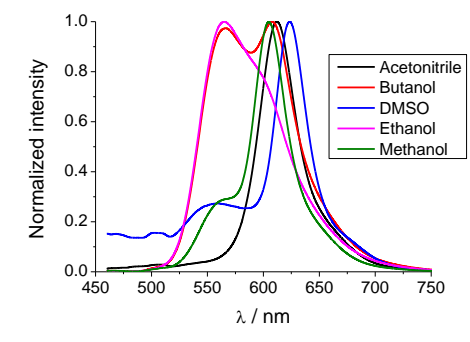 & 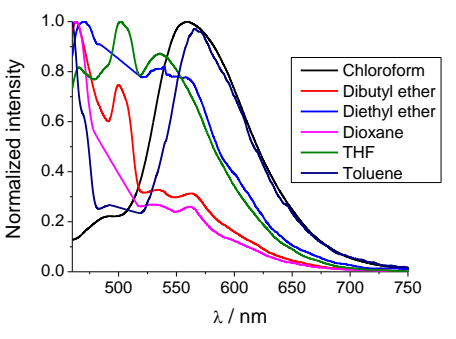 & 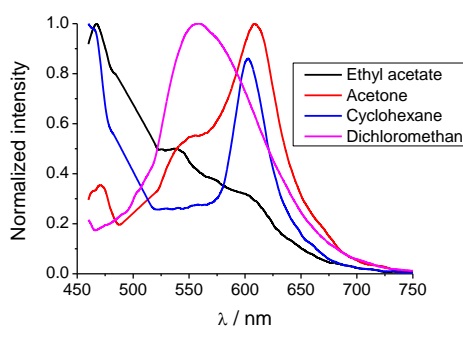 \\
\hline \multicolumn{3}{|c|}{$\begin{array}{l}\text { Figure A2: Normalized fluorescence emission spectra }\left(\lambda_{\mathrm{ex}}=440 \mathrm{~nm}\right) \text { of 2-Me-4-OMe-TM in } \\
\text { different solvents. The spectra are represented in separate panels for clarity. }\end{array}$} \\
\hline
\end{tabular}

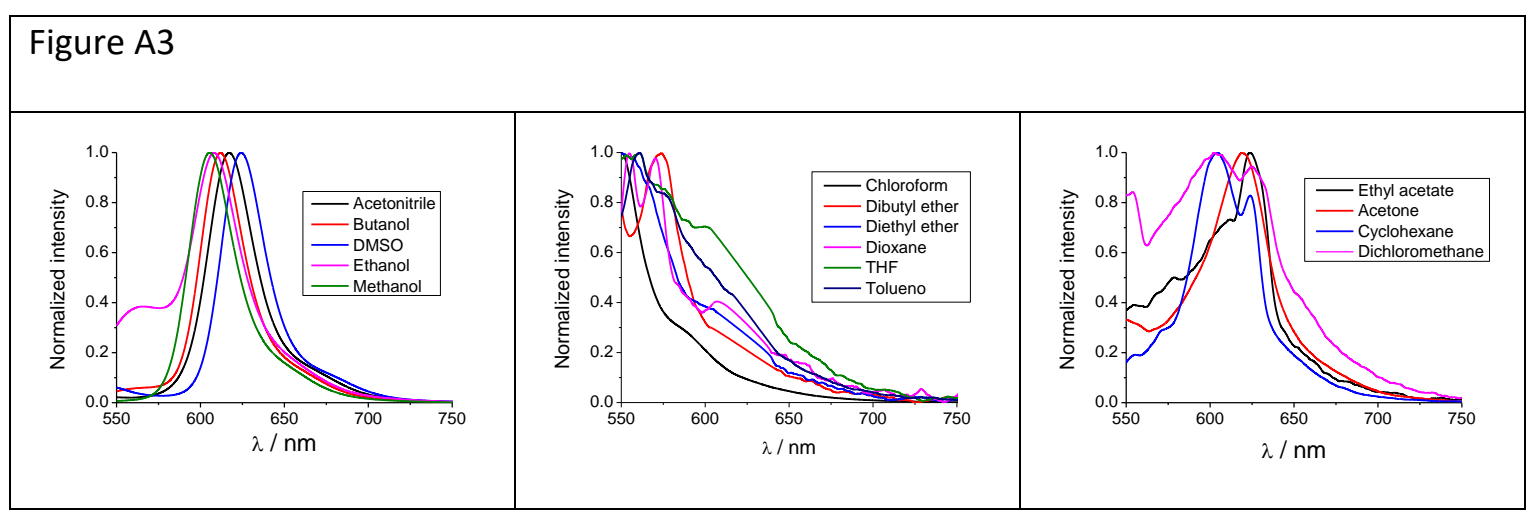


Figure A3: Normalized fluorescence emission spectra $\left(\lambda_{\mathrm{ex}}=530 \mathrm{~nm}\right)$ of 2-Me-4-OMe-TM in different solvents. The spectra are represented in separate panels for clarity.

\section{Figure A4}

A)

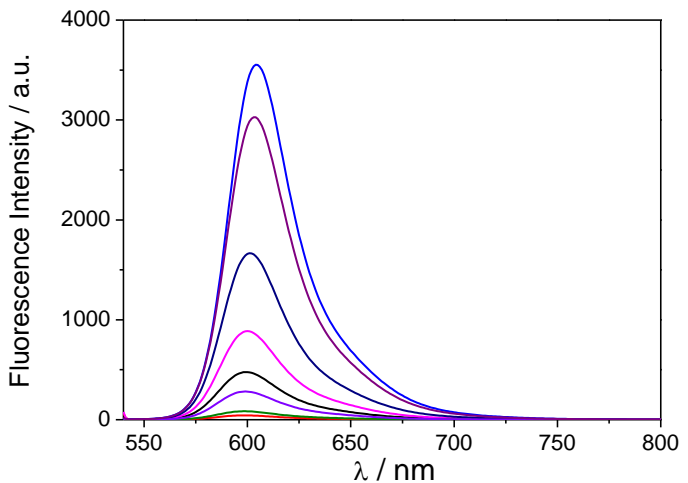

B)

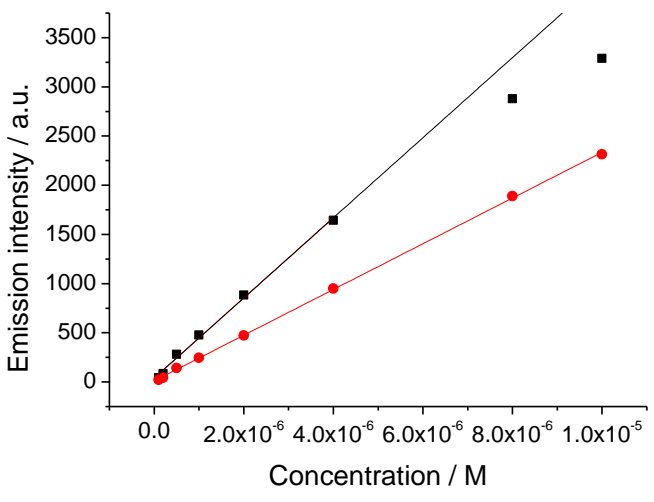

Figure A4: A) Fluorescence emission spectra_ $\left(\lambda_{\text {ex }}=530 \mathrm{~nm}\right)$ of different concentration of 2-Me4-OMe-TM (from $1 \times 10^{-7} \mathrm{M}$ until $1 \times 10^{-5} \mathrm{M}$ ) in aqueous solution. B) Kavanagh's Law at two different emission wavelengths (black squares $599 \mathrm{~nm}$ and red circles $620 \mathrm{~nm}$ )

\section{Figure A5}
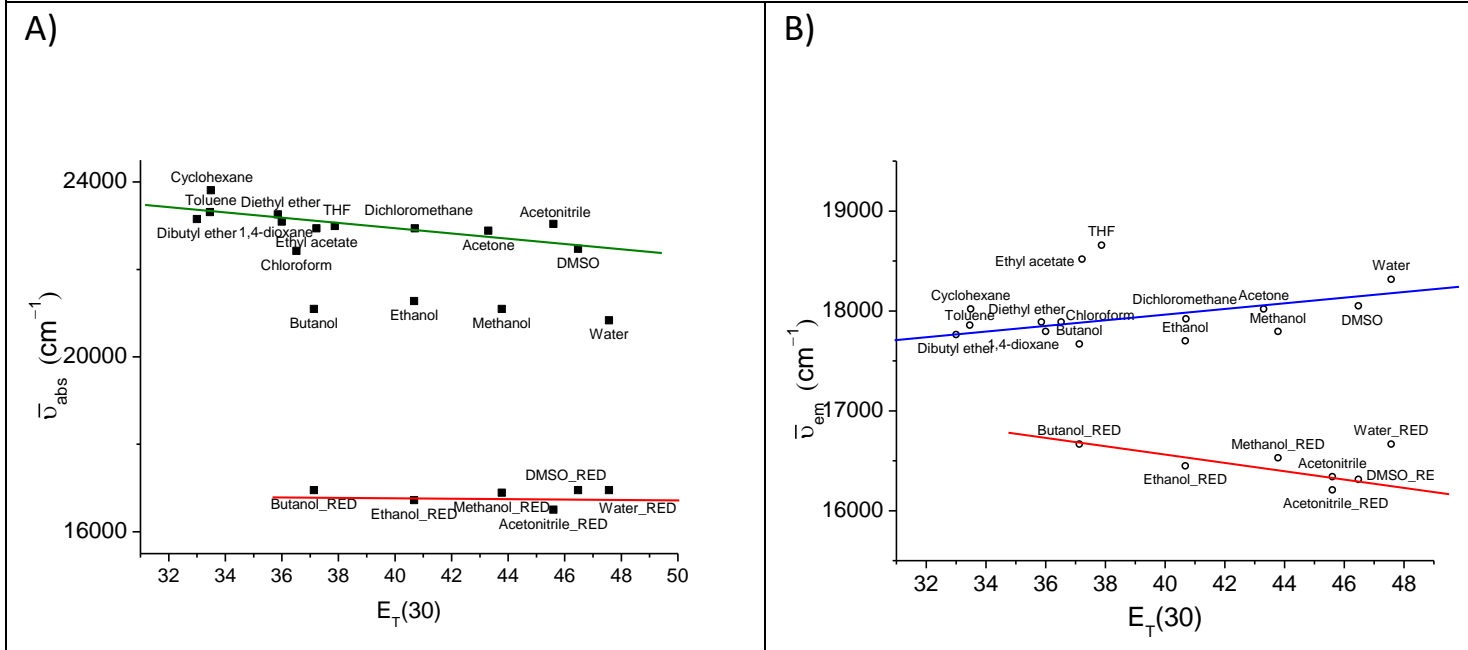

Figure A5: Plot of the absorption (A) and emission (B) maxima wavenumber as a function of the $\mathrm{E}_{\mathrm{T}}(30)$ parameter of the different solvents marked. Red lines and solvent with _RED 
inserted indicate the red-shifted band of the dye.

\begin{tabular}{|c|c|c|c|c|c|c|}
\hline \multicolumn{7}{|l|}{ Table A1 } \\
\hline \multirow[t]{2}{*}{ Solvent } & \multicolumn{2}{|c|}{$\lambda^{\max }{ }_{a b s} / n^{a}$} & \multicolumn{2}{|c|}{$\lambda^{\max }{ }_{e m} / n^{b}$} & \multicolumn{2}{|l|}{ QY } \\
\hline & & & $\left(\lambda_{\mathrm{ex}}=440 \mathrm{~nm}\right)$ & $\left(\lambda_{\mathrm{ex}}=530 \mathrm{~nm}\right)$ & 440 & 530 \\
\hline Acetone & 437 & - & 609 & 619 & $<0.001$ & - \\
\hline Acetonitrile & $434(100 \%)$ & $606(87 \%)$ & 612 & 617 & 0.007 & 0.009 \\
\hline Butanol & $474(17 \%)$ & $600(100 \%)$ & $566 / 608$ & 612 & 0.110 & 0.297 \\
\hline Cyclohexane & 420 & - & $460 / 602$ & 604 & 0.004 & - \\
\hline Chloroform & 446 & - & 559 & 550 & 0.011 & 0.023 \\
\hline Dibutylether & 432 & - & 464 & 574 & 0.001 & - \\
\hline Dichloromethane & 437 & - & 558 & 604 & 0.001 & - \\
\hline Diethylether & 430 & - & 469 & 552 & 0.001 & - \\
\hline Dioxane & 433 & - & 463 & 555 & 0.002 & - \\
\hline DMSO & $456(12 \%)$ & $613(100 \%)$ & 555 & 590 & 0.071 & 0.107 \\
\hline Ethyl acetate & 434 & - & 468 & 611 & 0.001 & - \\
\hline Ethanol & $470(100 \%)$ & $598(32 \%)$ & $565 /(\mathrm{s} 608)$ & $(565) / 608$ & 0.081 & 0.060 \\
\hline Methanol & $474(28 \%)$ & $592(100 \%)$ & (s 562)/605 & 605 & 0.058 & 0.267 \\
\hline THF & 435 & - & 535 & 557 & 0.005 & - \\
\hline Toluene & 429 & - & $460 / 567$ & 560 & 0.001 & - \\
\hline Water (aqNaOH) & - & 583 & 590 & 600 & & 0.44 \\
\hline \multicolumn{7}{|c|}{$\begin{array}{l}\text { aln solvents in which the dye exhibited two absorption bands, numbers in brackets represent the relative height of the two } \\
\text { peaks. }\end{array}$} \\
\hline
\end{tabular}




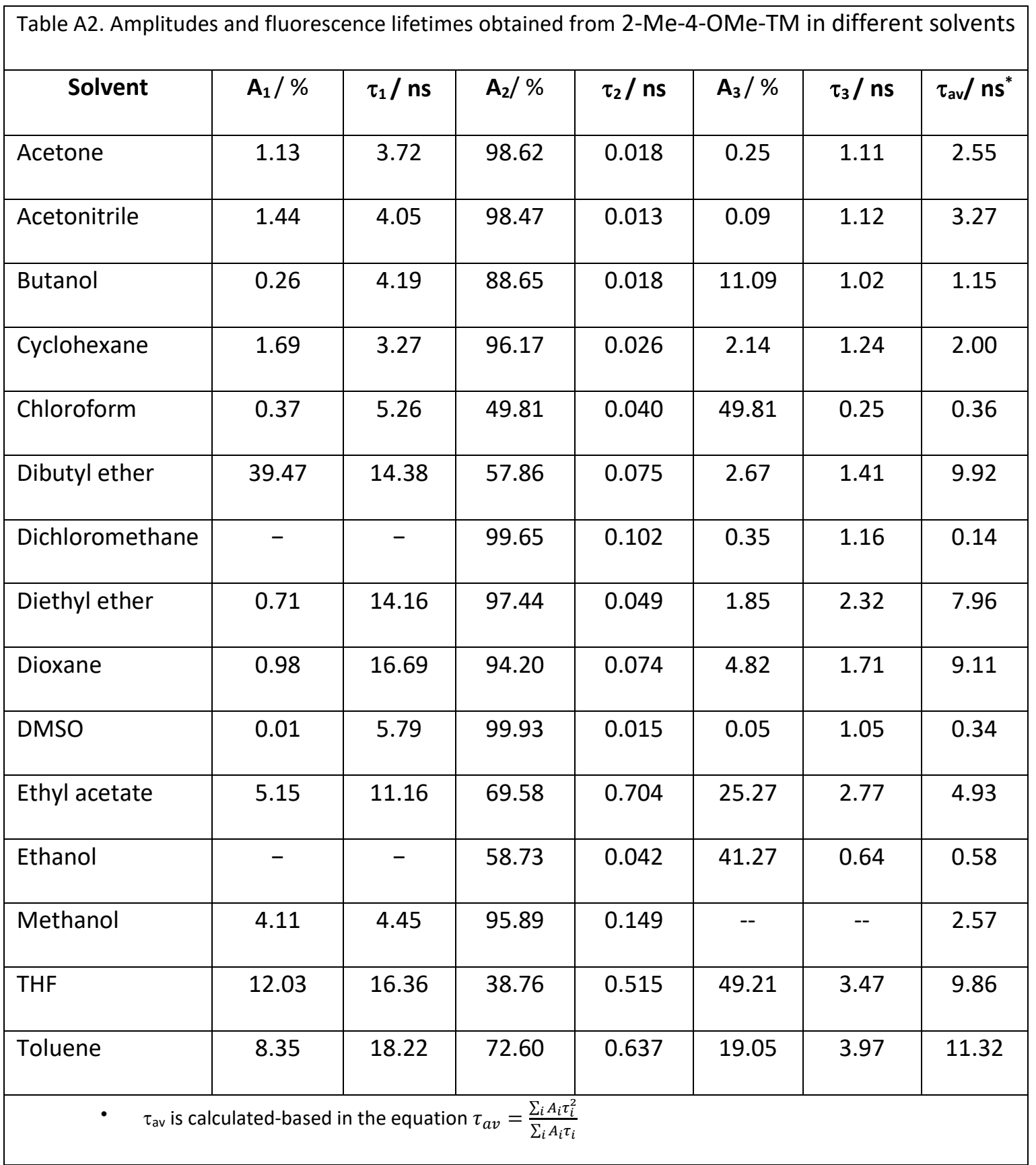




\begin{tabular}{|c|c|c|}
\hline Solvent & $\varepsilon$ & $n$ \\
\hline 1-Butanol & 17.8 & 1.399 \\
\hline Acetone & 21.01 & 1.359 \\
\hline Acetonitrile & 36.64 & 1.34423 \\
\hline Chloroform & 4.81 & 1.446 \\
\hline Cyclohexane & 2.02 & 1.426 \\
\hline Dibutyl ether & 3.1 & 1.399 \\
\hline Diethyl ether & 4.267 & 1.353 \\
\hline Dioxane & 2.21 & 1.422 \\
\hline DMSO & 47 & 1.358 \\
\hline Ethyl acetate & 5.99 & 6.02 \\
\hline Ethanol & 24 & 1.524 \\
\hline Methanol & 33 & 1.329 \\
\hline THF & 7.5 & 1.405 \\
\hline Toluene & 2.4 & 1.4961 \\
\hline Water & 80.1 & 1.33 \\
\hline \multicolumn{3}{|c|}{$\begin{array}{l}\text { *Refractive index used in this work come from the Refractive index } \\
\text { database (https://refractiveindex.info) and dielectric constant from }\end{array}$} \\
\hline
\end{tabular}


Lippert-Mataga equation

$$
\overline{v_{A}}-\overline{v_{F}}=\frac{2}{h c}\left(\frac{\varepsilon-1}{2 \varepsilon+1}-\frac{n^{2}-1}{2 n^{2}+1}\right) \frac{\left(\mu_{E}-\mu_{G}\right)^{2}}{a^{3}}+k
$$

In the Lippert-Mataga equation, $h$ is the Planck constant, $c$ represents the light speed in vacuum, $a$ is the radius of the cavity where the dye is allocated, $\overline{v_{A}}$ and $\overline{v_{F}}$ are the absorption and emission wavenumber, respectively, and $k$ is a constant representing the difference between the absorption and emission wavenumbers in the vacuum. Following this approach, the fluorophore is considered as a dipole plunged in a medium with equal dielectric constant at any point. The effects of $\varepsilon$ and $n$ on the Stokes shift can be explained as follows: an increase in $n$ produces an instantaneous stabilization of the ground and excited states due to an electronic redistribution producing a decrease in the difference of the energy between the ground and excited state. This effect is the reason why most of the chromophores show a redshift in the absorption spectra. However, although an increase in $\varepsilon$ also stabilizes both states, it occurs in the excited state after a reorientation of solvent dipoles, i.e., this process requires the movement of the complete solvent molecules. ${ }^{[3]}$ Orientation polarizability $(\Delta f)$ is the combination of both parameters as indicated in the equation and is included in the LippertMataga equation.

$$
\Delta f=\frac{\varepsilon-1}{2 \varepsilon+1}-\frac{n^{2}-1}{2 n^{2}+1}
$$




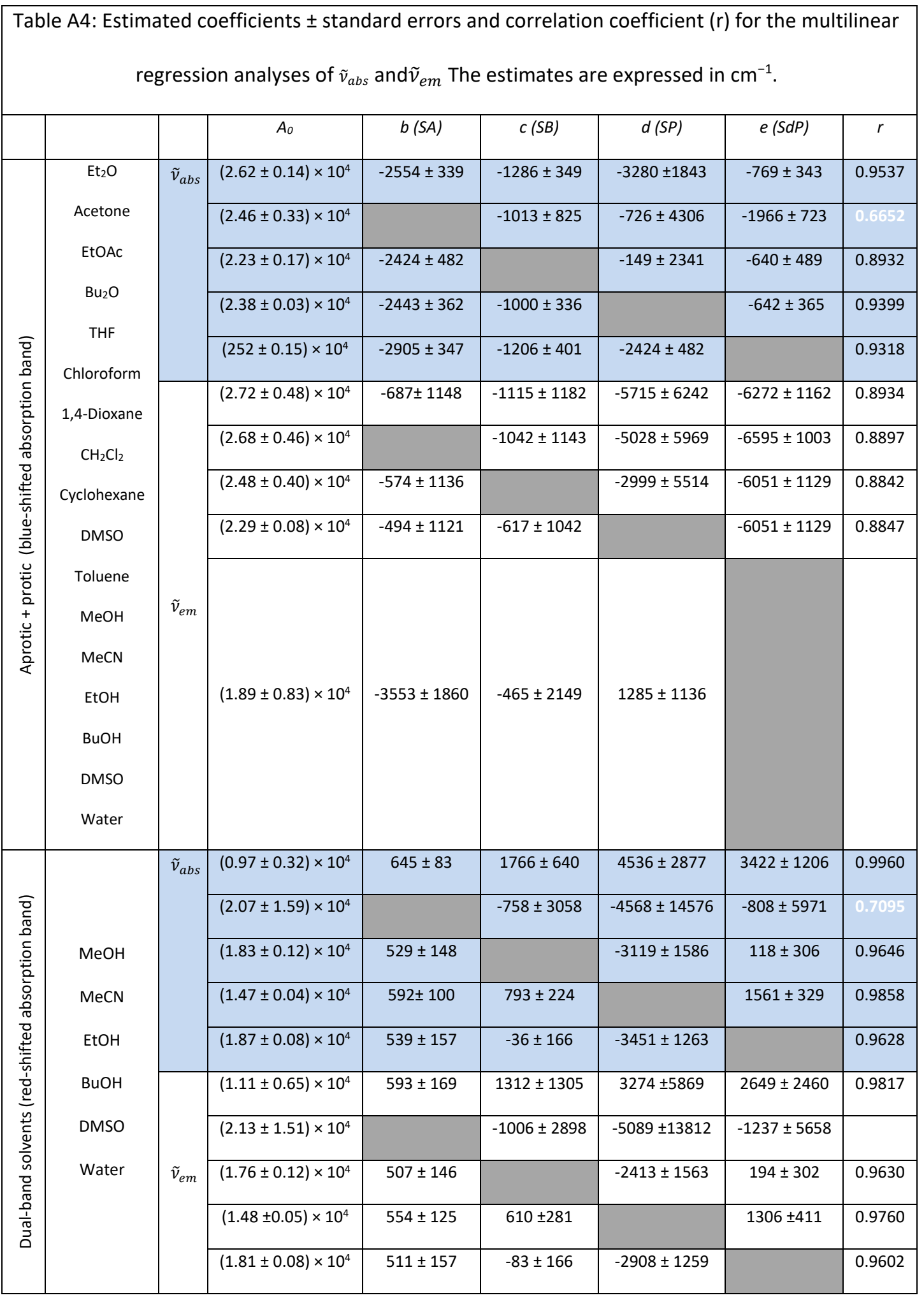




\section{Figure A6}

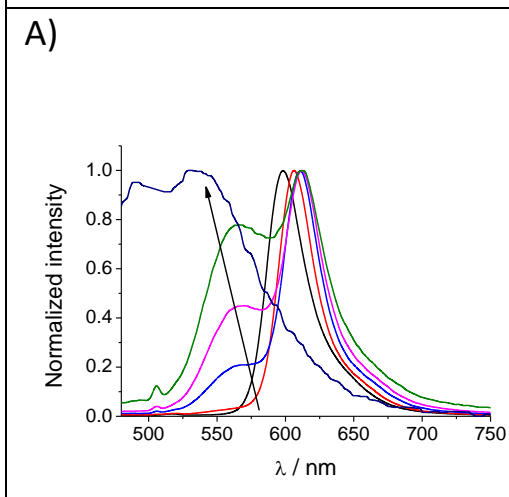

B)

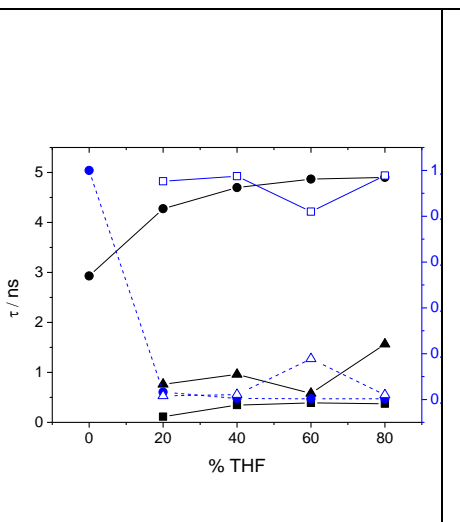

C)

Figure A6: Normalized intensity emission (A) at $\lambda_{\mathrm{ex}}=440 \mathrm{~nm}$ and fluorescence lifetimes $(\tau)$ and the relative abundances $(\rho)$ of 2-Me-4-OMe-TM in different water:THF proportions determined using B) $\lambda_{\mathrm{ex}}=440 \mathrm{~nm}$ and $\lambda_{\mathrm{em}}=570 \mathrm{~nm}$ and using C) $\lambda_{\mathrm{ex}}=440 \mathrm{~nm}$ and $\lambda_{\mathrm{em}}=600 \mathrm{~nm}$.

\section{$\underline{\text { Absorption studies }}$}

If a system that is formed by two species follows Beer's law, at any wavelength $\left(\lambda_{\mathrm{abs}}\right)$ and $\mathrm{pH}$, the absorbance $(A)$ is given by the expression

$$
A\left(p H, \lambda_{a b s}\right)=c\left(\sum_{i} \alpha_{i}\left(p H, p K_{a}\right) \varepsilon_{i}\left(\lambda_{a b s}\right)\right) d
$$

where $c$ is the total concentration of the dye, $d$ is the optical path length, $\varepsilon_{i}\left(\lambda_{a b s}\right)$ is the wavelength-dependent molar absorption coefficient of the ith prototropic form of the dye, and $\alpha_{i}\left(\mathrm{pH}, \mathrm{p} K_{a}\right)$ is the fraction of the dye in the ith prototropic form, which depends on both $\mathrm{pH}$ and $\mathrm{p} K_{\mathrm{a}}$.

$$
\begin{aligned}
& \alpha_{1}=\frac{\left[H^{+}\right]}{\left[H^{+}\right]+K_{a}} \\
& \alpha_{2}=\frac{K_{a}}{\left[H^{+}\right]+K_{a}}
\end{aligned}
$$

Substituting and operating properly, we obtain the next equation that is used to plot individual values versus $\mathrm{pH}$ to obtain the $\varepsilon_{N}$ and the $\mathrm{p} K_{a}$ 


$$
\frac{A\left(p H, \lambda_{a b s}\right)}{\varepsilon_{A} c}=\left(\frac{\varepsilon_{N}}{\varepsilon_{A}}\left(\lambda_{a b s}\right) \frac{10^{-p H}}{10^{-p H}+10^{-p k_{a}}}+\frac{10^{-p k_{a}}}{10^{-p H}+10^{-p k_{a}}}\right) d
$$

\section{Spectroscopy and photophysical properties of 2-Me-4-OMe-TM in aqueous solution and}

sensitivity towards phosphate ions

To understand the prototropic equilibrium of 2-Me-4-OMe-TM in aqueous solution, we studied the $\mathrm{pH}$ dependency through spectrophotometry and spectrofluorimetry around physiological $\mathrm{pH}$. The absorption spectra of 2-Me-4-OMe-TM at different $\mathrm{pH}$ values showed a similar shape as that of previously described silicon magenta xanthene dyes ${ }^{[2]}$ (see Figure A7). The presence of an isosbestic point at approximately $520 \mathrm{~nm}$ suggests the presence of two species. Using the fundamental equations of the chemical equilibrium and the Lambert-Beer law, we recovered the molar absorptivity coefficients of the two involved species, i.e., the neutral and anionic forms (see Scheme 2 and Figure A8), and a $p K_{a}$ value of $7.09 \pm 0.03$ (see Figure A9). At basic $p H$, the anionic form exhibited an absorption maximum at $583 \mathrm{~nm}$ and a shoulder at approximately $543 \mathrm{~nm}$. On the other hand, under acidic conditions, corresponding to the neutral form, the absorption was largely decreased and blue-shifted. The neutral species showed a broad and poorly defined absorption band centered at $480 \mathrm{~nm}$. Regarding the chemical equilibrium and using dyes of the same family as reference, ${ }^{[2 a, 7]}$ we propose scheme $A 2$ for the chemical equilibrium in aqueous solution, where a zwitterionic form is included. ${ }^{[7 b, 7 c, 8]}$ Interestingly, the fluorescence emission of this dye in aqueous solution is characterized by "on/off" properties, i.e., although the anionic form was very fluorescent, the neutral species presented a very low fluorescence emission in aqueous solution (see Figures A10, A11 and A12). 


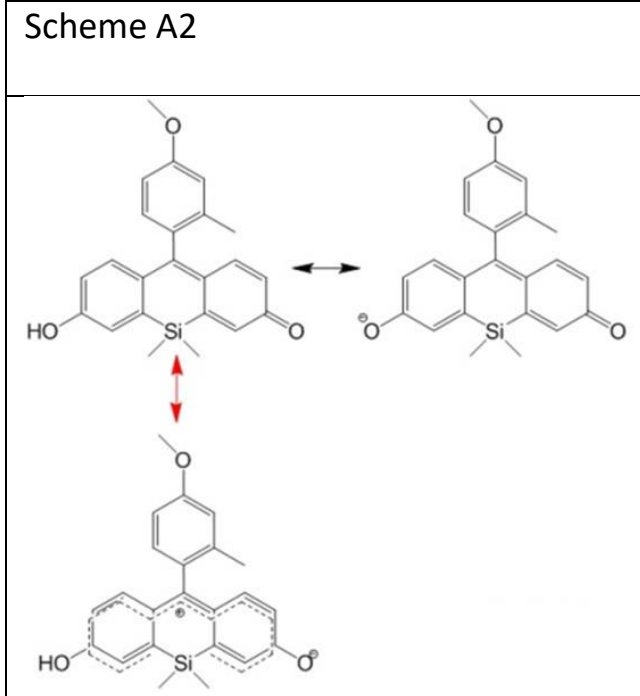

Scheme A2. Chemical equilibrium proposed between the prototropic neutral and anionic species of 2-Me-4-OMe-TM. Two resonance structures are included for the neutral species.

Similar to other xanthene derivatives, the presence of an adequate proton donor/acceptor can promote an ESPT reaction. As a consequence, the fluorescence decay times are dependent on the total phosphate concentration, so this mechanism has been used in the determination of intracellular phosphate concentration using FLIM imaging. ${ }^{[1,9]}$ For a better understanding of the phosphate sensitivity of this dye through the ESPT reaction, we have studied the excitedstate dynamics through time-resolved fluorescence spectroscopy. The basis of this study is well established $^{[10]}$ and can be resolved by measuring a data set of fluorescence decay traces at different $\mathrm{pH}$ values and buffer concentrations. Intracellular phosphate is an important parameter for many different physiological processes ${ }^{[11]}$; hence, since the species of the phosphate buffer can act as an adequate proton donor/acceptor pair, we have resolved the dynamics of the excited-state processes of 2-Me-4-OMe-TM in the presence of phosphate buffer. The scheme of the excited-state reactions and the corresponding rate constants is presented in Figure A13A. 
The analysis of the fluorescence decay traces of 2-Me-4-OMe-TM at different $\mathrm{pH}$ values and phosphate concentrations exhibited two decay times. The long decay time varied in the range of 2-3 ns, with an increasing trend as $\mathrm{pH}$ values increased and with lower values at higher phosphate concentrations (Fig. S11B). The short decay time varied between 80 and 200 ps, with less obvious trends, ultimately caused by the difficulty of accurately determining such short decay times. These decay time values can be fitted to the general equations of the buffer-mediated ESPT reaction, following the methodology previously described (see Appendix A, Buffer mediated ESPT reaction studies). Using the $\mathrm{pH}$ range in the experimental conditions, $k_{12}$ can be considered negligible because $\left[\mathrm{H}_{3} \mathrm{O}^{+}\right]$is small. Moreover, the shortest lifetime $\tau_{1}$ is the sum of the rate constant of the process that produces the disappearance of $1^{*}\left(k_{01}\right.$ and $\mathrm{k}_{21}$ ). We recovered the rate constants shown in Table $A 5$, which successfully describe the $\mathrm{pH}$ and phosphate dependency of the fluorescence decay times, as seen in the comparison of the estimated (lines) and experimental times (points) in Figure A13B. As can be observed, there is a very good concordance between these values. It is important to highlight that at physiological pH and below $400 \mathrm{mM}$ of phosphate, the fluorescence decay is characterized by a monoexponential character, which is a requirement for improved fluorescence lifetimebased sensors. Using $\mathrm{k}_{21}{ }^{\mathrm{B}}$ and $\mathrm{k}_{12}{ }^{\mathrm{B}}$, we obtained the excited-state $\mathrm{p} K_{a}{ }^{*}$ of the dye, ${ }^{[12]}$ resulting in a value of $6.85 \pm 0.08$. This $\mathrm{p} K_{a}{ }^{*}$ can be compared with that obtained through steady-state fluorescence measurements using a high phosphate concentration. Figure $\mathrm{A} 13 \mathrm{C}$ shows that the fit of the steady-state fluorescence data results in a $\mathrm{p} K_{a}{ }^{*}$ value of $6.58 \pm 0.06$. Although the dye is slightly more acidic in the excited state than in the ground state, as with other xanthene derivatives, ${ }^{[2 a, 7 b, 7 c]}$ the $\mathrm{p} K_{a}{ }^{*}$ is similar enough to the $\mathrm{p} K_{a}$ so that strong spontaneous ESPT reaction is negligible. 
Figure A7

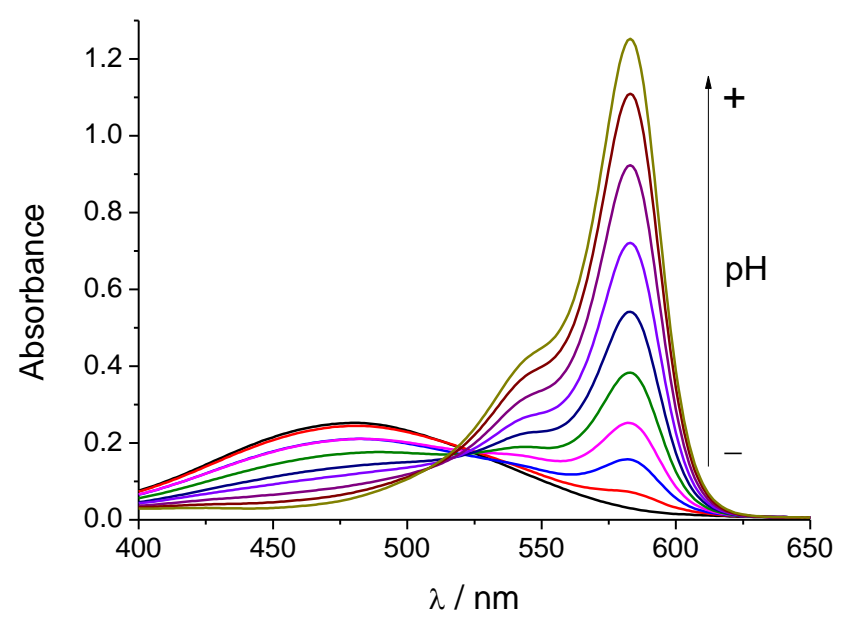

Figure A7: Absorption spectra of (at $4 \times 10^{-6} \mathrm{M}$ ) in phosphate buffer $0.01 \mathrm{M}$ at $\mathrm{pH}$ values between 4.88 and 8.94 . The arrow indicates increasing $\mathrm{pH}$ vales.

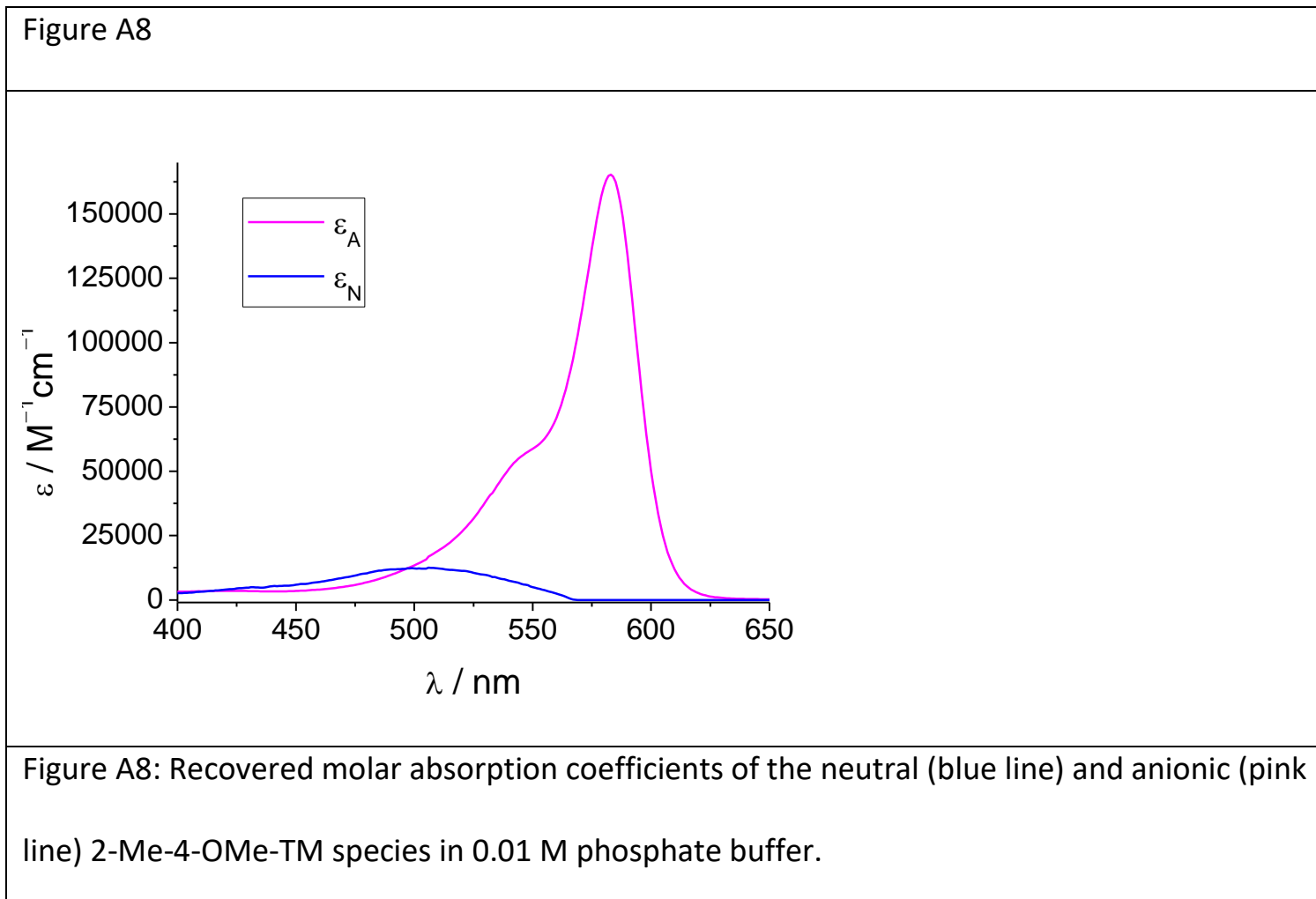




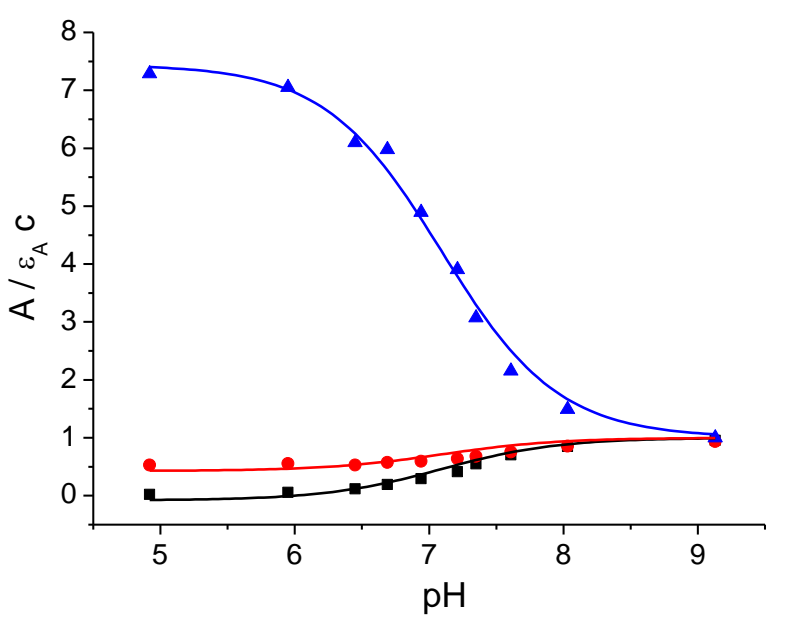

Figure A9: Global fitting of the $A /\left(\varepsilon_{A} C\right)$ at 440 (blue triangles), 530 (red circles) and 585 (black squares) $\mathrm{nm}$ versus $\mathrm{pH}$ curves of 2-Me-4-OMe-TM solutions in $0.01 \mathrm{M}$ phosphate buffer.

\section{Steady-state fluorescence studies}

The total fluorescence emission $F\left(\lambda_{\mathrm{ex}}, \lambda_{\mathrm{em}},\left[\mathrm{H}^{+}\right]\right)$at proton concentration $\left[\mathrm{H}^{+}\right]$produced by an excitation wavelength $\left(\lambda_{\text {ex }}\right)$ and observed at emission wavelength $\left(\lambda_{\text {em }}\right)$ can be expressed as:

$$
F\left(\lambda_{e x}, \lambda_{e m},\left[H^{+}\right]\right)=\frac{F_{\min }\left[H^{+}\right]+F_{\max } K_{a}}{K_{a}+\left[H^{+}\right]}
$$

where $F_{\min }$ indicates the fluorescence signal of the neutral form of the dye and $F_{\max }$ denotes the fluorescence signal of the anionic form of 2-Me-4-OMe-TM.

Quantum yield values from the steady-state fluorescence measurements were calculated using fluorescein in $0.1 \mathrm{M} \mathrm{NaOH}(\phi=0.79)^{[13]}$ and rhodamine 101 in ethanol $+0.01 \% \mathrm{HCl}(\phi=1.00)^{[14]}$ as references for the measurements for $\lambda_{\mathrm{ex}}=440 \mathrm{~nm}$ and $\lambda_{\mathrm{ex}}=530 \mathrm{~nm}$, respectively. 

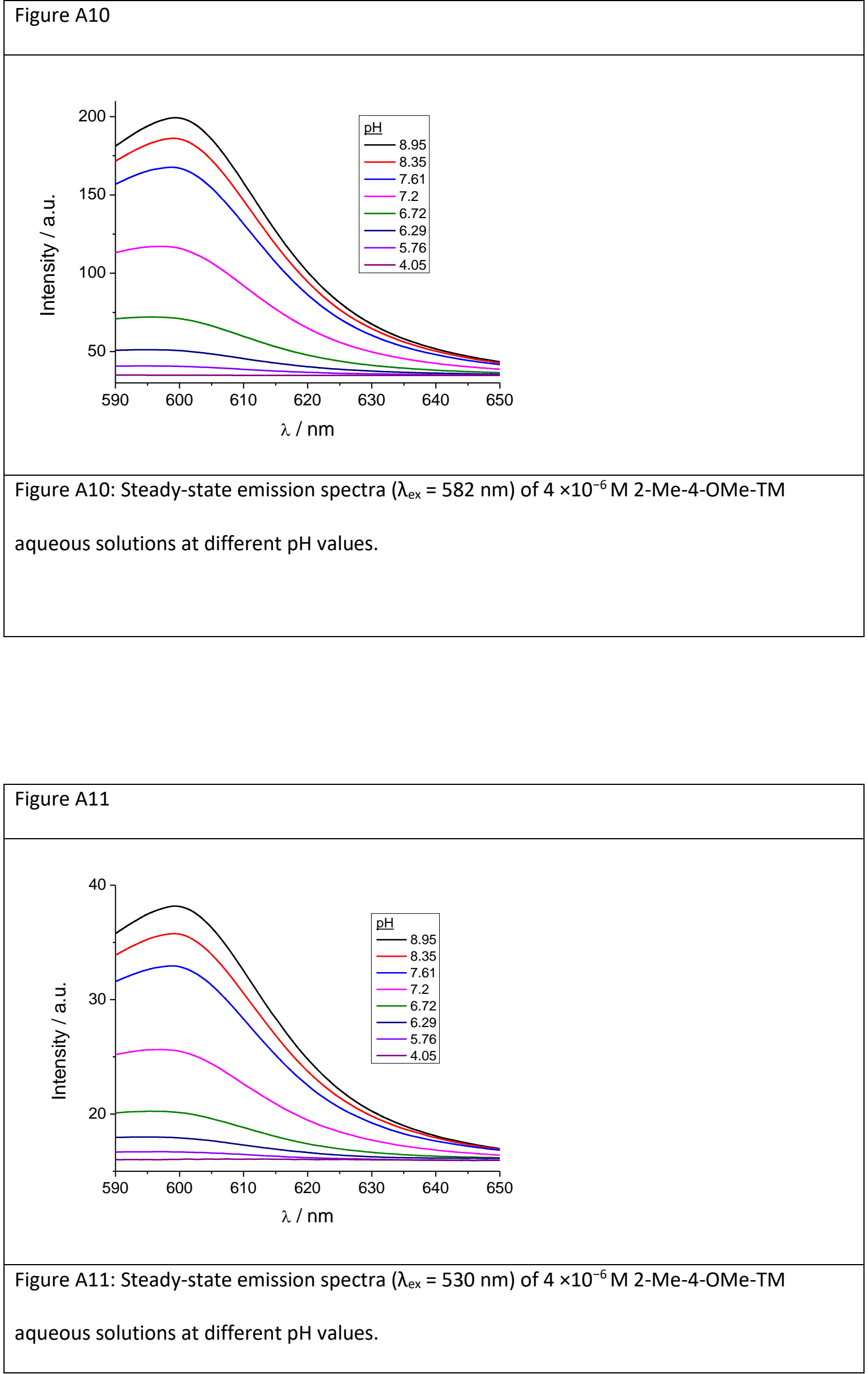


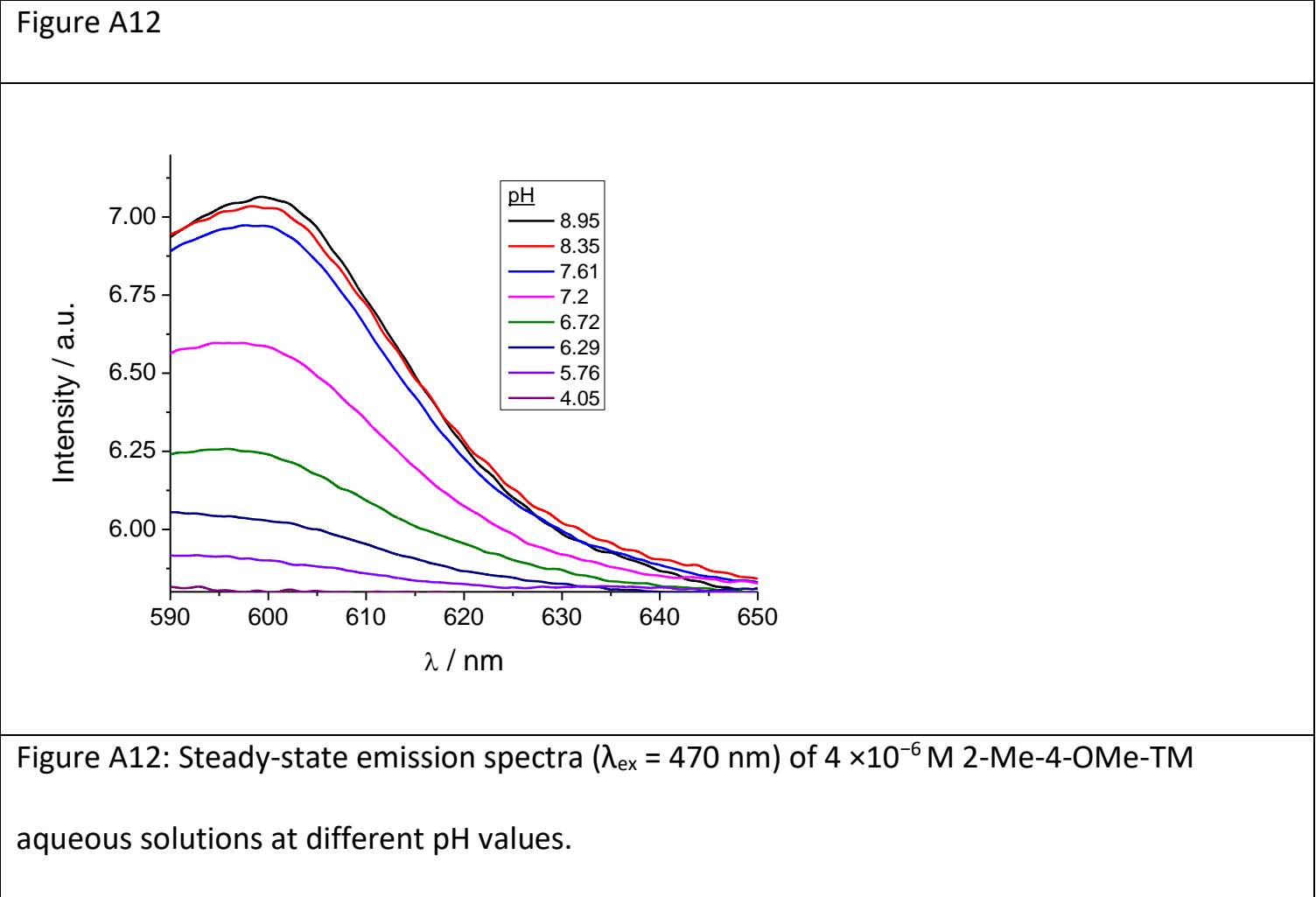

\section{Buffer-mediated ESPT reaction studies}

The theory and methods of solving buffer-mediated ESPT reactions are well established. ${ }^{[7 b, 10,}$

15]

Under experimental conditions where the buffer-mediated ESPT reaction occurs rapidly during its excited lifetime, the normalized fluorescence intensity can be expressed as ${ }^{[16]}$

$$
\frac{I}{A}=\frac{\phi_{1}}{1+10^{\left(p H-p K_{a}^{*}\right)}}+\frac{\phi_{2}}{1+10^{\left(p K_{a}^{*}-p H\right)}}
$$

where $\mathrm{I} / \mathrm{A}$ is the fluorescence intensity at $\lambda_{\mathrm{em}}$, normalized by absorbance at the excitation wavelength, $\phi_{1}$ and $\phi_{2}$ are the relative fluorescence efficiencies of the neutral and anionic forms at $\lambda_{\mathrm{em}}$, and $\mathrm{p} K_{a}{ }^{*}$ is the $\mathrm{p} K_{a}$ for the excited-state proton reaction $1^{*} \rightleftharpoons 2^{*}+\mathrm{H}^{+}$. 
If the photophysical system, as shown in Figure A3-A, is excited by an infinitely short light pulse that does not significantly alter the concentrations of the ground-state species, then the fluorescence $\delta$-response function, $f\left(\lambda_{\mathrm{em}}, \lambda_{\mathrm{ex}}, t\right)$, at emission wavelength $\lambda_{\mathrm{em}}$ due to excitation at $\lambda_{\text {ex }}$ is given by

$$
f\left(\lambda_{\text {ex }}, \lambda_{\text {em }}, t\right)=p_{1} e^{\gamma_{1} t}+p_{2} e^{\gamma_{2} t} \quad t \geq 0
$$

in which this equation has been written in the common biexponential character, where

$$
\begin{gathered}
\gamma_{1,2}=\frac{-(a+c) \pm \sqrt{(c-a)^{2}+4 b d}}{2} \\
a=k_{01}+k_{21}+k_{21}^{B}[R] \\
b=k_{12}\left[H^{+}\right]+k_{12}^{B}[R H] \\
c=k_{02}+k_{12}\left[H^{+}\right]+k_{12}^{B}[R H] \\
d=k_{21}+k_{21}^{B}[R]
\end{gathered}
$$

$[R]$ and $[R H]$ are the concentration of the basic and acidic prototropic forms of the buffer and are related to the total buffer concentration as $c^{B}=[R]+[R H]$. They can be expressed as $[R H]=$ $c^{B}\left[H^{+}\right] /\left(\left[H^{+}\right]+K_{a}^{B}\right)$ and $[R]=C^{B} K_{a}^{B} /\left(\left[H^{+}\right]+K_{a}^{B}\right)$, where $K_{a}^{B}$ is the dissociation constant for the reversible reaction $R H \rightleftharpoons R+H^{+} . \mathrm{k}_{01}, \mathrm{k}_{02}, \mathrm{k}_{12}, \mathrm{k}_{21}, \mathrm{k}^{\mathrm{B}}{ }_{12}$ and $\mathrm{k}_{21}^{\mathrm{B}}$ are the kinetic constants shown in Figure $1 \mathrm{~A}$.

The $\gamma$ factors are related to the fluorescence lifetimes $\tau_{1}$ and $\tau_{2}$ by the expression

$$
\tau_{1,2}=-\frac{1}{\gamma_{1,2}}
$$


A)
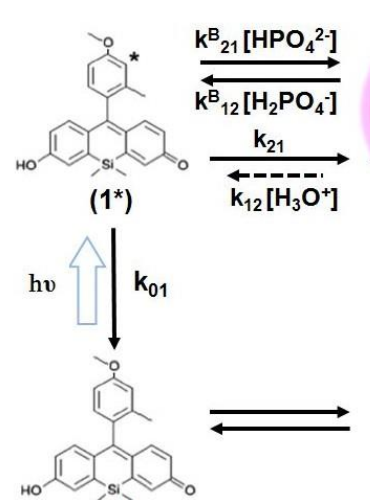

(1)
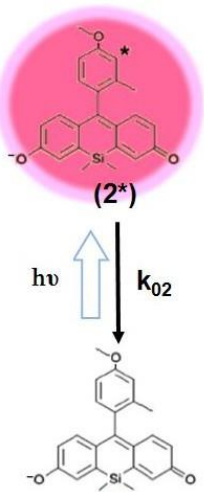

(2)
B)

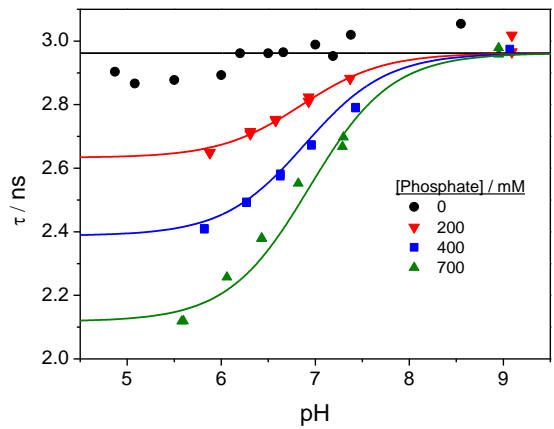

C)

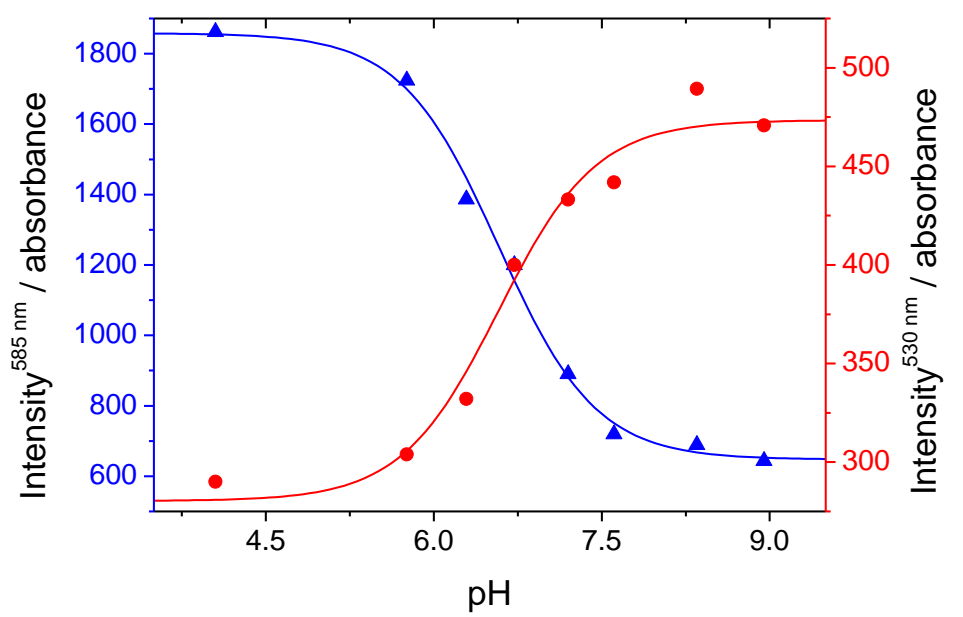

Figure A13. A) Proposed kinetic model of ground- and excited-state proton-transfer reactions of 2-Me-4-OMe-TM in the presence of phosphate buffer. B) Global fitting (solid lines) of the theoretical equations (see Appendix $\mathrm{A}$ ) to the long decay time at different $\mathrm{pH}$ values and phosphate buffer concentrations (circles: 0, invert triangles: 200, squares: 400 and triangles: $700 \mathrm{mM}$ ). C) Global normalized fluorescence emission plot vs $\mathrm{pH}$ of 2-Me-4-OMe-TM in phosphate buffer solution ( $0.7 \mathrm{M}$ ) at 585 (blue triangles) and 530 (red circles) $\mathrm{nm}$. The lines represent the global fit used to determine the $\mathrm{p} K_{a}{ }^{*}$. 


\begin{tabular}{|c|c|}
\hline \multicolumn{2}{|c|}{ Table A5 } \\
\hline Rate constants & Value \\
\hline $\boldsymbol{k}_{\mathbf{0 1}}\left(\boldsymbol{s}^{\mathbf{- 1}}\right)$ & $1.33( \pm 0.89) \times 10^{9}$ \\
\hline $\boldsymbol{k}_{\mathbf{0 2}}\left(\boldsymbol{s}^{\mathbf{- 1}}\right)$ & $3.37( \pm 0.01) \times 10^{8}$ \\
\hline $\boldsymbol{k}_{\mathbf{2 1}}\left(\boldsymbol{s}^{\mathbf{- 1}}\right)$ & $7.82( \pm 0.92) \times 10^{9}$ \\
\hline $\boldsymbol{k}_{\mathbf{2 1}}^{\boldsymbol{B}}\left(\boldsymbol{M}^{\mathbf{- 1}} \boldsymbol{s}^{\mathbf{1}}\right)$ & $2.03( \pm 1.34) \times 10^{9}$ \\
\hline $\boldsymbol{k}_{\mathbf{1 2}}^{\boldsymbol{B}}\left(\boldsymbol{M}^{\mathbf{- 1}} \boldsymbol{s}^{\mathbf{- 1}}\right)$ & $1.95( \pm 0.99) \times 10^{9}$ \\
\hline
\end{tabular}




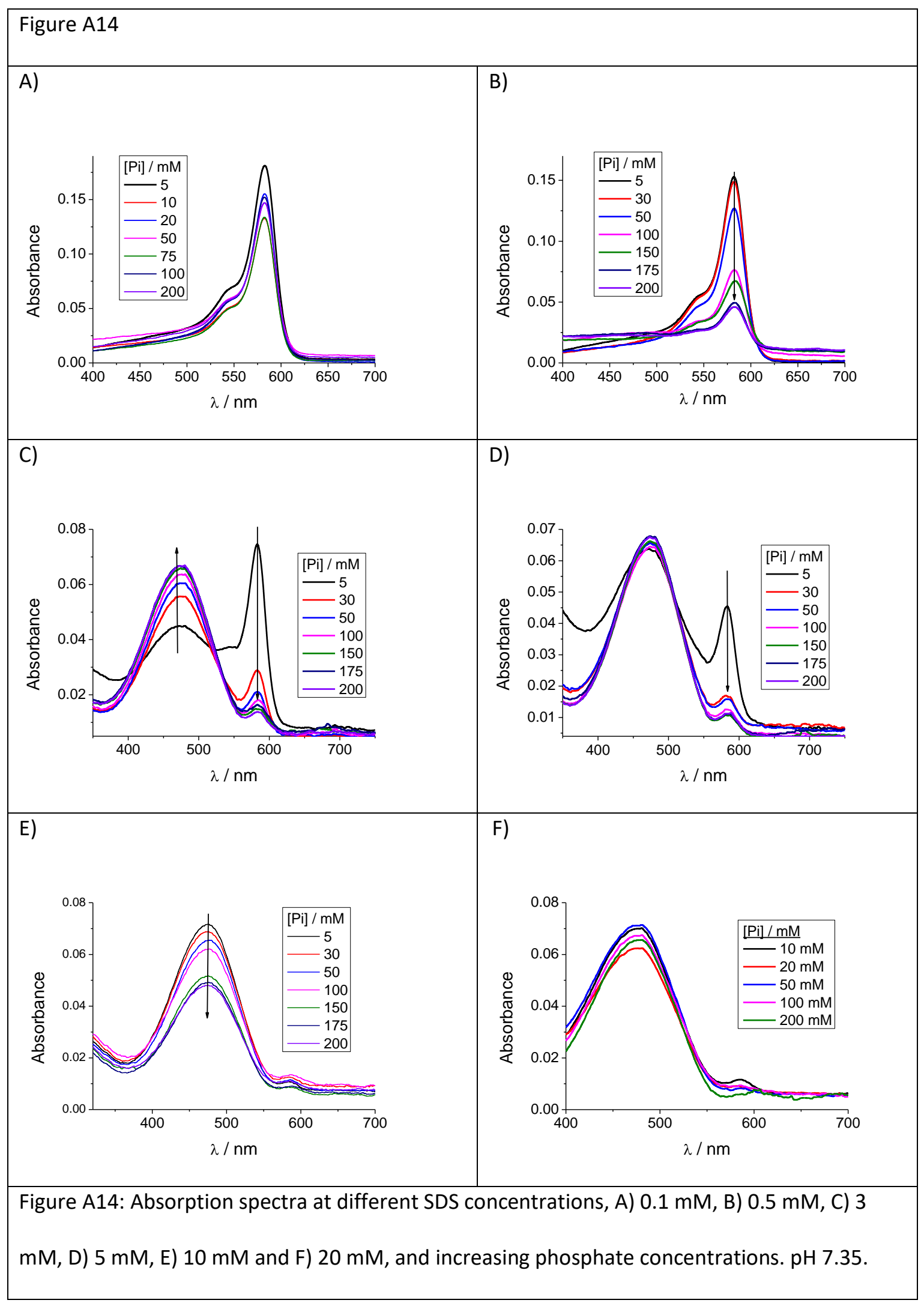




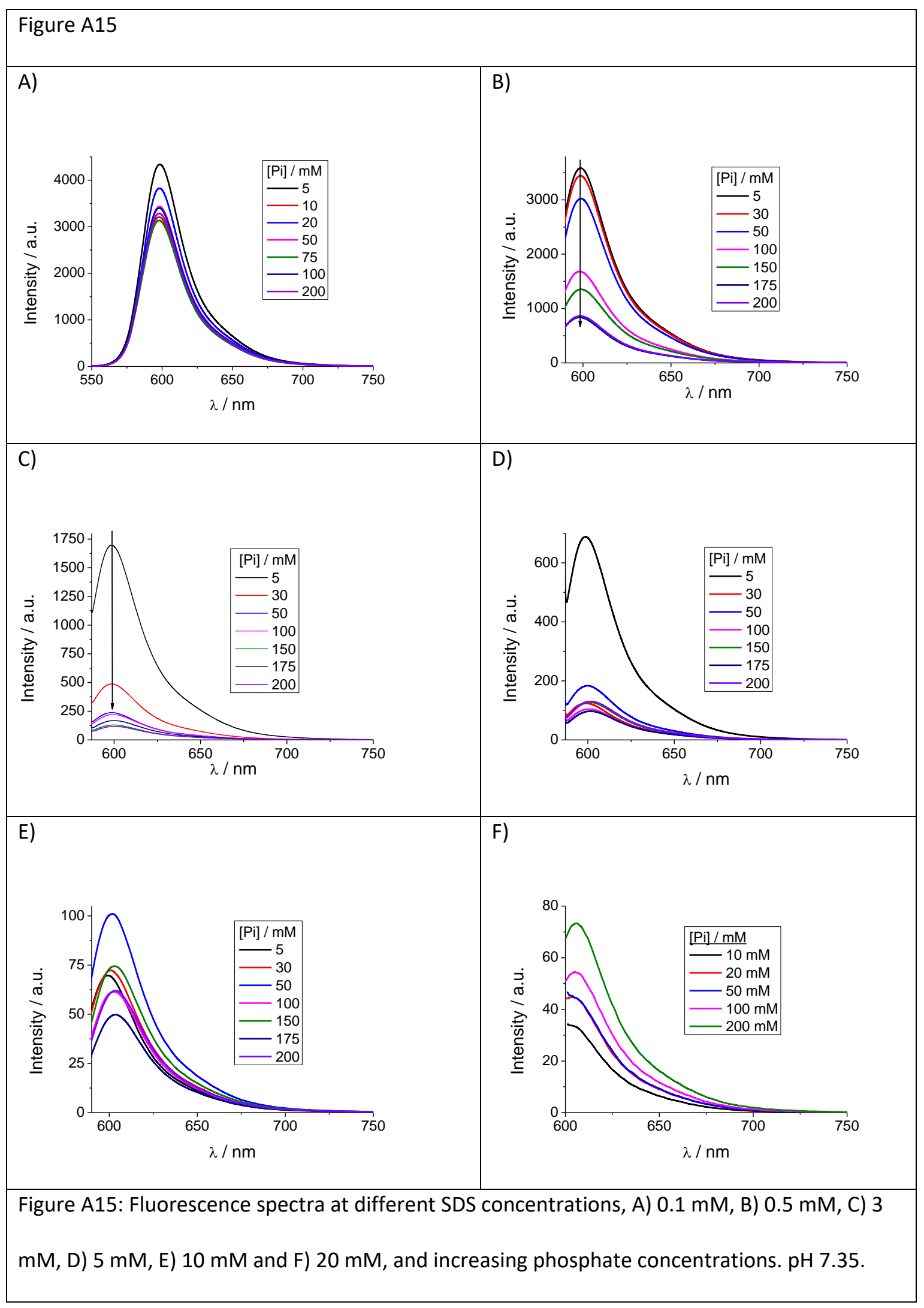




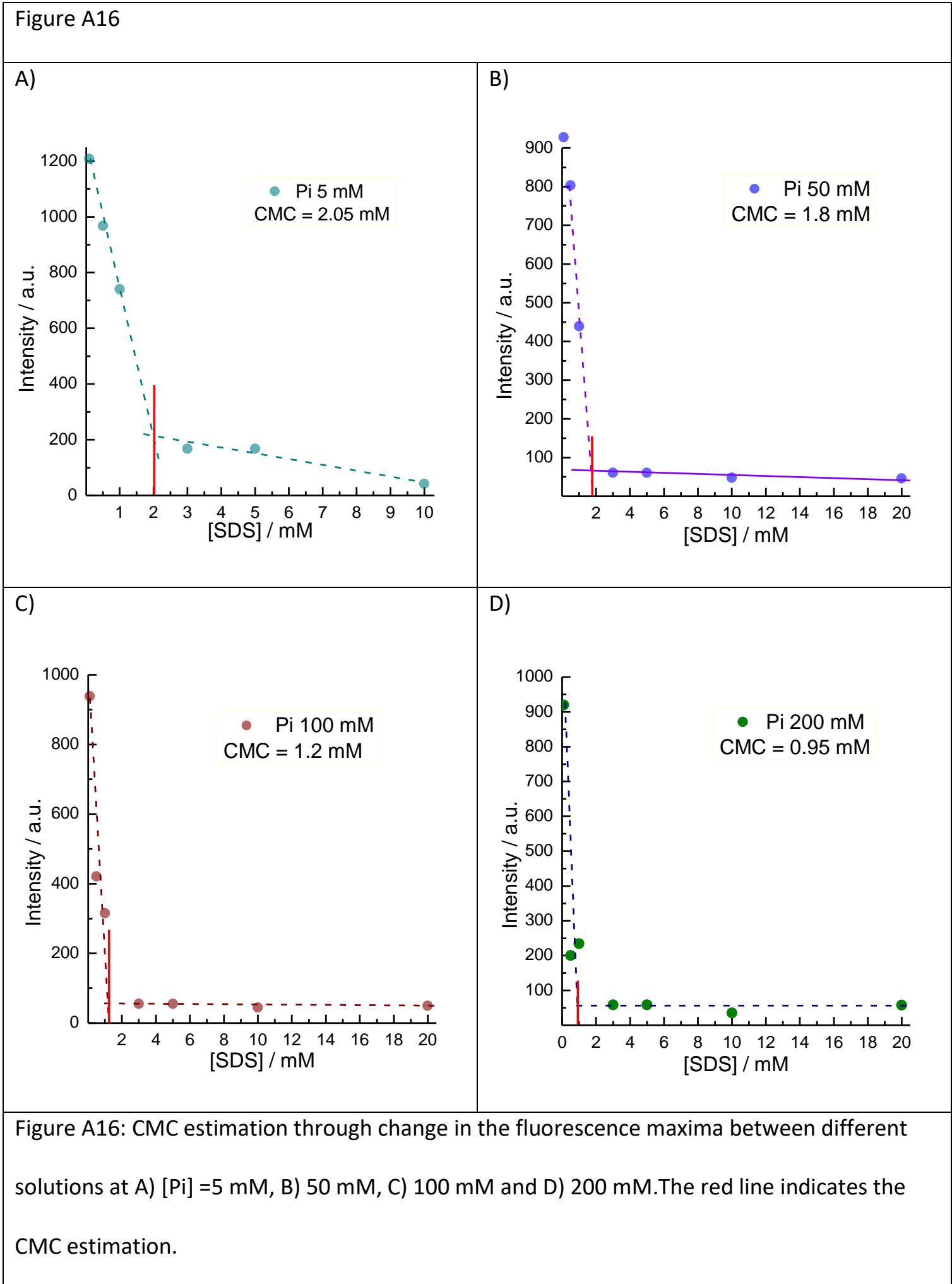




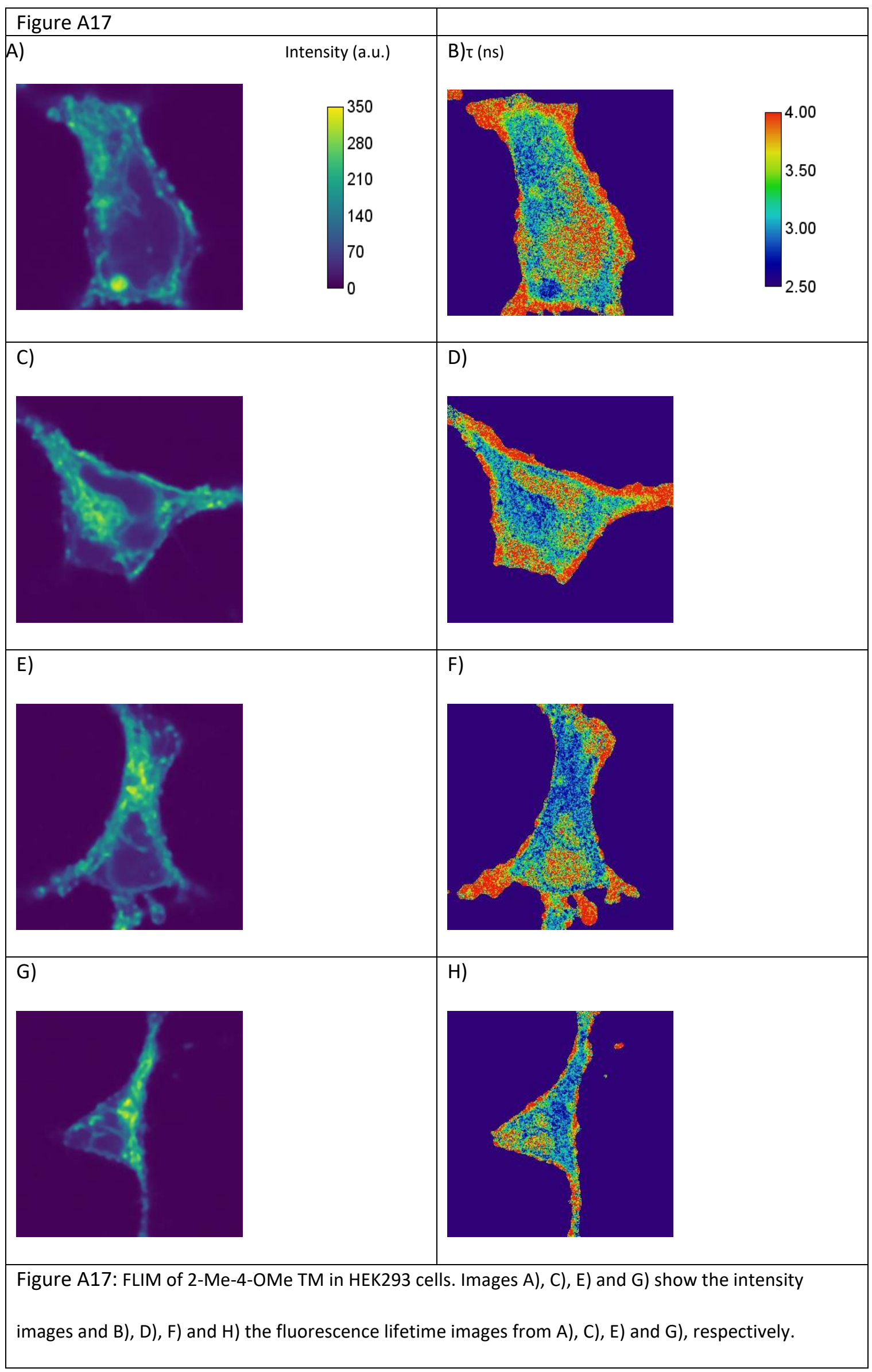




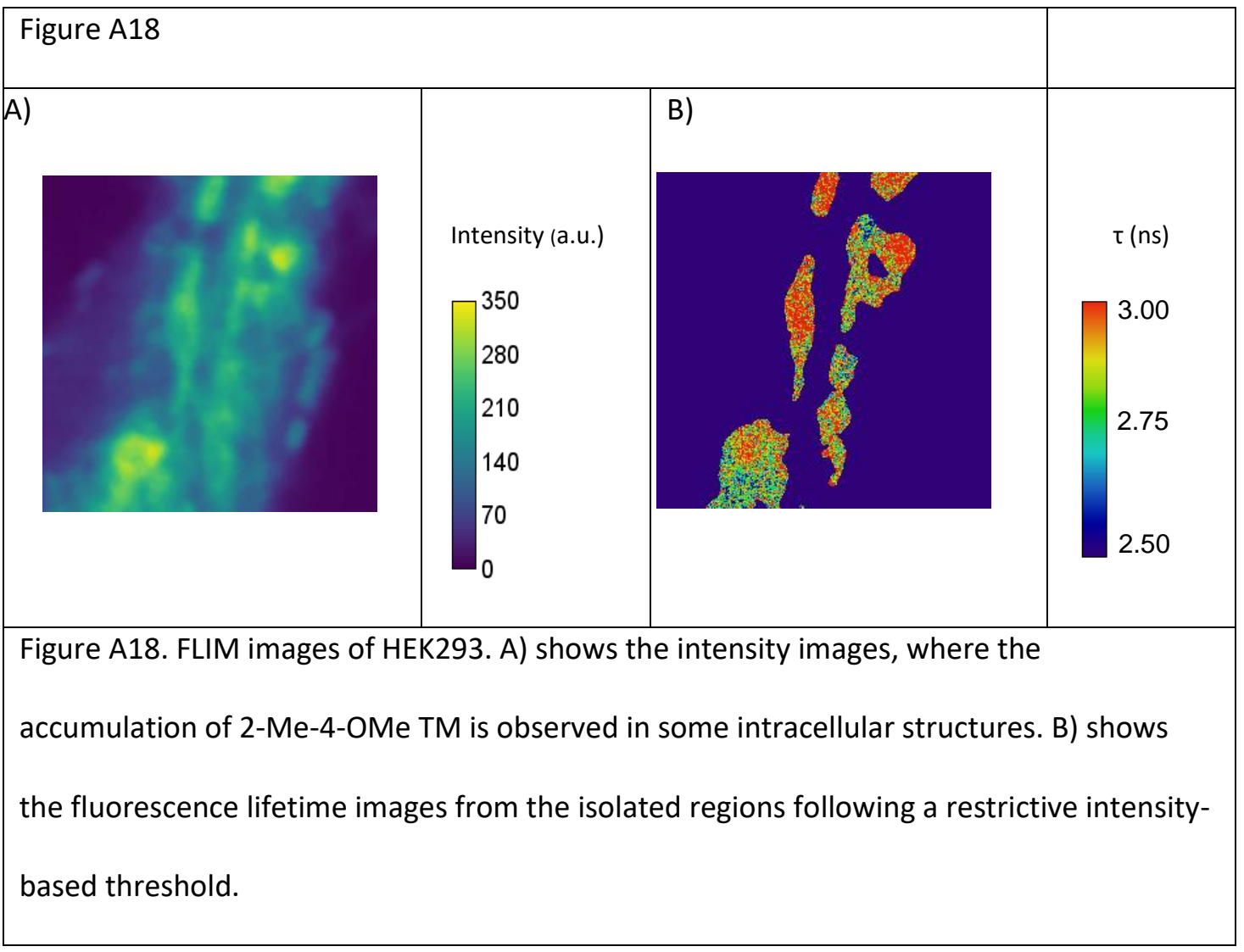

\section{$\underline{\text { References }}$}

[1] P. Herrero-Foncubierta, J. M. Paredes, M. D. Giron, R. Salto, J. M. Cuerva, D. Miguel, A. Orte, Sensors 2018, 18, 18.

[2] a) L. Crovetto, A. Orte, J. M. Paredes, S. Resa, J. Valverde, F. Castello, D. Miguel, J. M. Cuerva, E. M. Talavera, J. M. Alvarez-Pez, Journal of Physical Chemistry A 2015, 119, 10854-10862; b) Q. A. Best, N. Sattenapally, D. J. Dyer, C. N. Scott, M. E. McCarroll, Journal of the American Chemical Society 2013, 135, 13365-13370.

[3] J. R. Lakowicz, Principles of Fluorescence Spectroscopy, 3rd ed., Springer, 2006.

[4] L. Porres, A. Holland, L. O. Palsson, A. P. Monkman, C. Kemp, A. Beeby, Journal of Fluorescence 2006, 16, 267-272.

[5] J. Karpiuk, Z. R. Grabowski, F. C. Deschryver, Journal of Physical Chemistry 1994, 98, 3247-3256.

[6] C. Wohlfarth, Static Dielectric Constants of Pure Liquids and Binary Liquid Mixtures, Springer, Berlin, Heidelberg, 2015.

[7] a) V. Puente-Munoz, J. M. Paredes, S. Resa, A. M. Ortuno, E. M. Talavera, D. Miguel, J. M. Cuerva, L. Crovetto, Sensors and Actuators B-Chemical 2017, 250, 623-628; b) L. Crovetto, J. M. Paredes, R. Rios, E. M. Talavera, J. M. Alvarez-Pez, Journal of Physical Chemistry A 2007, 111, 13311-13320; c) J. M. Paredes, L. Crovetto, R. Rios, A. Orte, J. M. Alvarez-Pez, E. M. Talavera, Physical Chemistry Chemical Physiscs 2009, 11, 54005407.

[8] A. Orte, M. J. Ruedas-Rama, J. M. Paredes, L. Crovetto, J. M. Alvarez-Pez, Langmuir 2011, 27, 12792-12799. 
[9] Q. Zhang, K. Xiao, J. M. Paredes, T. Mamonova, W. B. Sneddon, H. Liu, D. Wang, S. Li, J. C. McGarvey, D. Uehling, R. Al-awar, B. Joseph, F. Jean-Alphonse, A. Orte, P. A. Friedman, Journal of Biological Chemistry 2019.

[10] L. Crovetto, A. Orte, E. M. Talavera, J. M. Alvarez-Pez, M. Cotlet, J. Thielemans, F. C. De Schryver, N. Boens, Journal of Physical Chemistry B 2004, 108, 6082-6092.

[11] J. M. Paredes, M. D. Giron, M. J. Ruedas-Rama, A. Orte, L. Crovetto, E. M. Talavera, R. Salto, J. M. Alvarez-Pez, Journal of Physical Chemistry B 2013, 117, 8143-8149.

[12] A. Orte, L. Crovetto, E. M. Talavera, N. Boens, J. M. Alvarez-Pez, Journal of Physical Chemistry A 2005, 109, 734-747.

[13] J. Q. Umberger, V. K. LaMer, Journal of the American Chemical Society 1945, 67, 10991109.

[14] T. Karstens, K. Kobs, The Journal of Physical Chemistry 1980, 84, 1871-1872.

[15] a) J. M. Alvarez-Pez, L. Ballesteros, E. Talavera, J. Yguerabide, Journal of Physical Chemistry A 2001, 105, 6320-6332; b) A. Martinez-Peragon, D. Miguel, R. Jurado, J. Justicia, J. M. Alvarez-Pez, J. M. Cuerva, L. Crovetto, Chemistry 2014, 20, 447-455.

[16] J. Yguerabide, E. Talavera, J. M. Alvarez, B. Quintero, Photochemistry and Photobiology 1994, 60, 435-441. 